\title{
Investigating the normal state and superconducting state properties of orthorhombic and hexagonal ZrRuP: A first-principles study
}

\author{
S. Bağc1 $\odot,{ }^{1}$ M. Cin, ${ }^{1}$ H. Y. Uzunok,,${ }^{1,2}$ Ertuğrul Karaca, ${ }^{2}$ H. M. Tütüncü $\odot,{ }^{1,2}$ and G. P. Srivastava ${ }^{3}$ \\ ${ }^{1}$ Sakarya Üniversitesi, Fen-Edebiyat Fakültesi, Fizik Bölümü, 54187, Sakarya, Turkey \\ ${ }^{2}$ Sakarya Üniversitesi, BIMAYAM Biyomedikal, Manyetik ve Yarililetken Malzemeler Araştırma Merkezi, 54187, Sakarya, Turkey \\ ${ }^{3}$ School of Physics, University of Exeter, Stocker Road, Exeter EX4 4QL, United Kingdom
}

(Received 5 September 2019; published 7 November 2019)

\begin{abstract}
We have executed $a b$ initio pseudopotential calculations on the structural, electronic, elastic, mechanical, vibrational, and electron-phonon interaction properties of hexagonal $\mathrm{ZrRuP}(\mathrm{h}-\mathrm{ZrRuP}$ ) and orthorhombic $\mathrm{ZrRuP}$ (o-ZrRuP). The electronic states of both phases near the Fermi energy are found to be dominated by the $d$ electrons of transition metal atoms, suggesting that they play a more active role in the generation of superconducting state for both phases of $\mathrm{ZrRuP}$. A critical assessment of their elastic and mechanical properties reveals that the lattice of $\mathrm{h}-\mathrm{ZrRuP}$ is softer than that of o-ZrRuP. A comparison of phonon dispersion curves for both phases indicates that the lower transverse acoustic branch of h-ZrRuP is much softer than that of $\mathrm{o}-\mathrm{ZrRuP}$. The soft character of this phonon branch gives rise to strong electron-phonon interaction in h-ZrRuP. Therefore the electron-phonon coupling parameter for h-ZrRuP equals to 1.25 which is considerably larger than the corresponding value of 0.57 for o-ZrRuP. As a consequence, phonon and electron-phonon interaction properties are crucial in making superconducting transition temperature much higher for h-ZrRuP than o-ZrRuP. At the end, the value of this temperature is found to be $12.49 \mathrm{~K}$ for h-ZrRuP and $3.89 \mathrm{~K}$ for o-ZrRuP which coincide with their experimental values of 12.93 and $3.82 \mathrm{~K}$.
\end{abstract}

DOI: 10.1103/PhysRevB.100.184507

\section{INTRODUCTION}

Equiatomic intermetallic $T T^{\prime} X$ compounds ( $T$ : electronpoor transition metal, $T^{\prime}$ : electron-rich transition metal, $X$ : $\mathrm{P}$, As, and $\mathrm{Si}$ ) have been widely investigated in the least 50 years with respect to their interesting structural [1-13] and magnetic [14-26] properties. Related to the size and the electron count of the transition metal, these compounds either exist in the orthorhombic TiNiSi-type structure [1] or the hexagonal $\mathrm{ZrNiAl}$-type structure [3]. Both structures are favorable for the occurrence of superconductivity and thus the main interest in the $T T^{\prime} X$ compounds has concerned their superconducting behavior [27-47]. ZrRuP is one important member of intermetallic $T T^{\prime} X$ superconductors since it exhibits polymorphism and can adopt either the hexagonal ZrNiAl-type structure (h-ZrRuP) [27,29] or the orthorhombic TiNiSi-type structure (o-ZrRuP) [30,31,33]. The phase transition from the orthorhombic phase to the hexagonal one for $\mathrm{ZrRuP}$ occurs at around $3.5 \mathrm{GPa}$ and $1100^{\circ} \mathrm{C}$ [33]. h-ZrRuP has the highest superconducting transition temperature $\left(T_{c}\right)$ of $13 \mathrm{~K}$ [39] among the hexagonal ZrNiAl-type superconductors. On the other hand, the $T_{c}$ value of o-ZrRuP has been reported to be around $4 \mathrm{~K}$ [30], which is much lower than that of the hexagonal phase. It has been always questioned why $\mathrm{h}$-ZrRuP possesses a much higher $T_{c}$ than does o-ZrRuP. Barz and co-workers [27] have proposed that the high $T_{c}$ value of $\mathrm{h}-\mathrm{ZrRuP}$ can be associated with the short $\mathrm{Ru}-\mathrm{Ru}$ interatomic distance of $2.63 \AA$, in it which is shorter than the corresponding distance value of $2.68 \AA$ in the Ru metal. This reduction in the value of $\mathrm{Ru}-\mathrm{Ru}$ distance may decreases the value of the $4 d$ bandwidth and thus increases the value of density of states at the Fermi level $\left(N\left(E_{F}\right)\right)$ for h-ZrRuP. As a consequence, the high $T_{c}$ value of h-ZrRuP may supposably arise from its high $N\left(E_{F}\right)$ value [27]. However, the specific heat measurement of $\mathrm{h}-\mathrm{ZrRuP}$ [28] reveals that its $N\left(E_{F}\right)$ possesses a relatively low value of 0.76 states/eV per atom which is even $30 \%$ lower than that for the Ru metal. This experimental observation totally withers the suggestion made by Barz and co-workers [27]. The specific heat measurement of o-ZrRuP has been also realized from 2 to $15 \mathrm{~K}$ [31]. From this measurement, the value of $N\left(E_{F}\right)$ for o-ZrRuP is found to be 0.72 states/eV per atom which is almost identical to that for h-ZrRuP. This observation proposes that the large $T_{c}$ difference between $\mathrm{h}-\mathrm{ZrRuP}$ and o-ZrRuP can not be explained in terms of their $N\left(E_{F}\right)$ values. Although both phases have similar $N\left(E_{F}\right)$ values, their Debye temperatures $[28,31]$ $\left(\Theta_{D}^{\mathrm{h}-Z \mathrm{rRuP}}=345 \mathrm{~K}\right.$ and $\left.\Theta_{D}^{\mathrm{o}-\mathrm{ZrRuP}}=454 \mathrm{~K}\right)$ differ from each other within $30 \%$. Therefore the $30 \%$ softer lattice of h-ZrRuP as compared to that of o- $\mathrm{ZrRuP}$ may be responsible for its high $T_{c}$ value since soft phonon modes may enhance the value of electron-phonon coupling parameter for $\mathrm{h}-\mathrm{ZrRuP}$ and thus increase its $T_{c}$ value. The confirmation of this suggestion certainly requires phonon calculations on the both phases of ZrRuP, but unfortunately they have been totally ignored in the literature so far. Shirotani and co-workers [34] have explored that $\mathrm{h}-\mathrm{ZrRuSi}$ exhibits superconducting transition at around $12 \mathrm{~K}$ although the closest $\mathrm{Ru}-\mathrm{Ru}$ distance in this superconductor is $9 \%$ longer than that in $\mathrm{h}-\mathrm{ZrRuP}$. This observation indicates that the $T_{c}$ of these superconductors is insusceptible to the $\mathrm{Ru}-\mathrm{Ru}$ distance in contrast with the suggestion made by 
Barz and co-workers [27]. Shirotani and co-workers [36-40] have compared the superconducting parameters, the number of valence electrons and the cell volumes per formula unit for h-HfRuP, h-ZrRuP, h-ZrRuAs, h-ZrRuSi, o-ZrRuGe, and o-NbPS in their experimental studies. This comparison reveals that these compounds exhibit the superconducting transition above $10 \mathrm{~K}$ although their cell volume are different from each other. Therefore they have concluded that the superconductivity of these equiatomic compounds insusceptible to their cell volume. Furthermore, the phosphides and arsenide have 17 valance electrons and this number is 16 for the silicide but their $T_{c}$ values are almost equal to each other. This observation [36-40] reveals that the superconductivity of these compounds are not susceptive to the number of valance electrons although the electrical properties of many compounds depend crucially on the number of valance electrons. All above experimental studies on the both phases of $\mathrm{ZrRuP}$ signal that a sound explanation of the large $T_{c}$ difference between h-ZrRuP and o-ZrRuP certainly requires phonons and electron-phonon interaction calculations on these phases which have been totally ignored in the literature.

The occurrence of superconductivity in the both phases of $\mathrm{ZrRuP}$ promoted theoreticians to study their structural and electronic properties while their elastic, vibrational and electron-phonon interaction properties have been not investigated yet. Seo and co-workers [48] have realized the extended Huckel tight-binding (EHTB) band structure calculations on the both phases of ZrRuP. Their calculations [48] produce the value of $N\left(E_{F}\right)$ to be 0.21 and 0.29 states/eV per atom for $\mathrm{h}-\mathrm{ZrRuP}$ and o-ZrRuP, respectively. Despite these values are substantially lower than their corresponding experimental values attained by the specific heat measurements of both phases [28,31], this theoretical work supports that only electronic structure calculations are not enough to explain the large $T_{c}$ difference between these phases due to their similar $N\left(E_{F}\right)$ values. The electronic properties of $\mathrm{h}-\mathrm{ZrRuP}$ and oZrRuP have been analyzed by using the full-potential linearized augmented plane wave (FLAPW) method within the local density approximation (LDA) $[49,50]$. These theoretical studies reveal that the bands of both phases near the Fermi level are mostly due to $d$-orbital character. These theoretical works give the value of $N\left(E_{F}\right)$ to be 0.71 states/eV per atom for both phases. Since both phases have the equal $N\left(E_{F}\right)$ value, their phonon properties must play a noteworthy role in the generation of their superconducting states. In addition to these theoretical calculations [48-50], the electronic structures of h-ZrRuP and o-ZrRuP have been computed using the orthogonalized linear combination of atomic orbitals method (OLCAO) based on density functional theory with its LDA [51]. In agreement with previous theoretical calculations [48-50], this theoretical work [51] supports that the electronic states of both phases close to the Fermi level are mainly dominated by the $d$ states of transition metal atoms while the contributions to the value of $N\left(E_{F}\right)$ from the $\mathrm{P}$ atoms are very small. Although the structural and electronic properties of both phases have presented and discussed, phonon and electron-phonon interaction calculations on them are completely lacking from the literature. However, for the metallic system, many physical properties, such as electrical and thermal resistivity, thermal expansion, and superconductivity, appear to be governed by phonons and their interactions with electrons. The aim of the present work is to probe the structural, electronic, elastic, mechanical, phonon and electron-phonon interaction properties of $\mathrm{h}-\mathrm{ZrRuP}$ and $\mathrm{o}-\mathrm{ZrRuP}$ in order to bring a prominent explanation on the large $T_{c}$ difference between these phases.

In this paper, we have targeted to view the structural and electronic properties of h-ZrRuP and o-ZrRuP by using the density functional theory with its generalized gradient approximation (GGA) [52,53]. The structural and electronic properties of both phases are exhibited and discussed in detail. Then, the efficient stress-strain method calculations [54,55] have been realized in order to specify the second order elastic constants of both phases and the calculated results suggest the mechanical stability of $\mathrm{ZrRuP}$ in its hexagonal and orthorhombic structures. After obtaining second order elastic constants, the values of polycrystalline bulk modulus (B), shear modulus $(\mathrm{G})$, Young's modulus (E), and Poisson's ratio $(\sigma)$ for both phases have been calculated using the VoigtReuss-Hill (VHR) approach [56-58]. Then, our structural and electronic results have been used to investigate phonon properties of h-ZrRuP and o-ZrRuP by the help of the linear response method [52,53]. Our phonon results signal the dynamical stability of ZrRuP in its hexagonal and orthorhombic structures. After phonon calculations, the linear response approach [52,53] and the Migdal-Eliashberg approach $[59,60]$ have been combined to calculate electron-phonon matrix elements for both phases of ZrRuP. Finally, our phonon results and electron-phonon matrix elements have been utilized to calculate the Eliashberg spectral functions of both phases which enables us to obtain their average electron-phonon coupling parameter and logarithmic average of phonon frequency.

\section{METHOD}

All calculations have been performed by the first-principles calculations based on the density functional theory implemented in the QUANTUM ESPRESSO code $[52,53]$. The solution of Kohn-Sham equations [61] have been executed making use of Perdew-Burke-Ernzerhof GGA [62]. Norm-conserving pseudopotentials [63] are used to model the electron-ion interactions. Throughout our calculations, a plane-wave cutoff energy of $60 \mathrm{Ry}$ is used to determine the number of plane waves in expansion. The both phases of $\mathrm{ZrRuP}$ have been fully relaxed by making use of the Broyden-Fletcher-GoldfrabShanno optimized method [64]. Momentum space integration has been realized by utilizing the Monkhorst-Pack special $\boldsymbol{k}$ points sampling scheme over the irreducible part of the Brillouin zone (IBZ) [65]. Total energy calculations for hZrRuP have been conducted by using a $(8 \times 8 \times 8)$ zonecentered grid while a $(6 \times 6 \times 6)$ zone-centered grid is used for $\mathrm{o}-\mathrm{ZrRuP}$. However, the electronic calculations for both phases have been executed by using a denser $(24 \times 24 \times 24)$ zone-centered grid.

The linear response approach calculations [52,53] have been executed in order to study phonons in the both phases of $\mathrm{ZrRuP}$. We have used a $(8 \times 8 \times 8)$ zone-centered grid and a $(6 \times 6 \times 6)$ zone-centered grid for sampling the irreducible segment of the Brillouin zone (BZ) in our phonon calculations for h-ZrRuP and o-ZrRuP, respectively. We have 
directly calculated a total of twelve dynamical matrices for h-ZrRuP on a $4 \times 4 \times 4 \boldsymbol{q}$-point mesh. For o-ZrRuP, we have computed a total of eight dynamical matrices on a $2 \times 2 \times 2$ $\boldsymbol{q}$-point mesh. Finally, these dynamical matrices have been Fourier transformed to procure the full phonon spectrum and density of states. We have combined the linear-response approach [52,53] and the Migdal-Eliashberg theory [59,60] in order to study electron-phonon interaction in the both phases of ZrRuP. The details of electron-phonon interaction calculations have been clearly explained in our previous studies [66] and thus will not be repeated here. However, we have to mention that the Fermi surface sampling for the evaluating of the electron-phonon interaction matrix elements have been conducted by making use of a denser $(24 \times 24 \times 24)-k$ mesh.

\section{RESULTS}

\section{A. Structural and electronic properties}

The hexagonal and orthorhombic crystal structures of $\mathrm{ZrRuP}$ are illustrated in Fig. 1. As can be seen from this

(a)
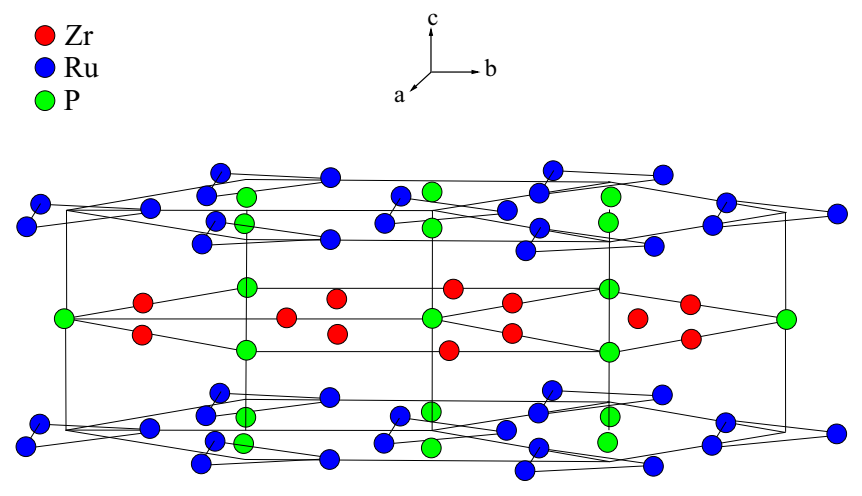

(b)
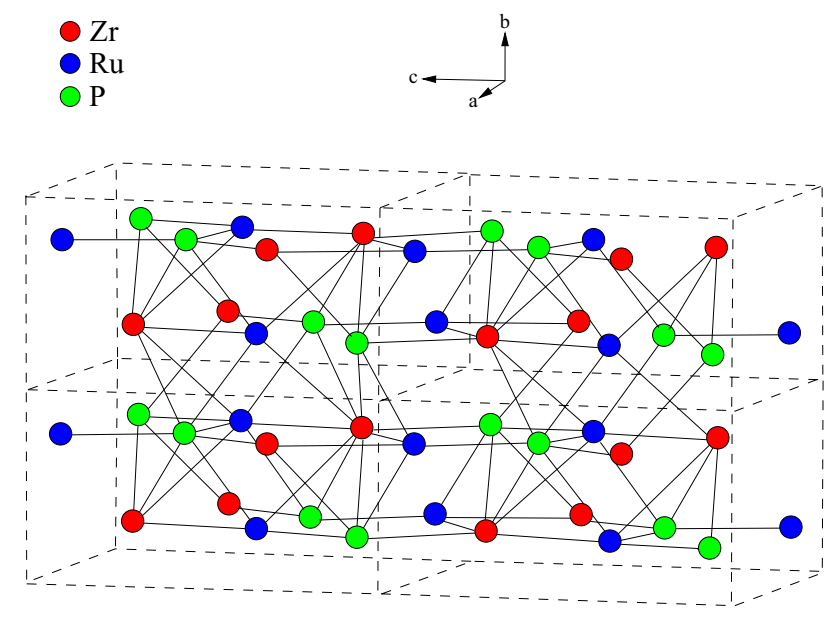

FIG. 1. (a) The layer crystal structure of h-ZrRuP with a hexagonal $\mathrm{ZrNiAl}$-type form. Either $\mathrm{Zr}$ and $\mathrm{P}$ atoms or $\mathrm{Ru}$ and $\mathrm{P}$ atoms occupy each layer in the hexagonal lattice. (b) The layer crystal structure of o-ZrRuP with an orthorhombic TiNiSi-type form. On the contrary, the orthorhombic phase contains layers which are filled with $\mathrm{Zr}$, $\mathrm{Ru}$, and $\mathrm{P}$ atoms and thus all layers are equivalent. figure, both phases of $\mathrm{ZrRuP}$ are layer structures. Each layer in the hexagonal phase contains either $\mathrm{Zr}$ and $\mathrm{P}$ atoms or $\mathrm{Ru}$ and $\mathrm{P}$ atoms. On the contrary, the orthorhombic phase is formed by layers which are filled with $\mathrm{Zr}, \mathrm{Ru}$, and $\mathrm{P}$ atoms. Therefore different from the hexagonal phase, all layers are equivalent in the orthorhombic phase. Being isostructural with the well-known $\mathrm{ZrNiAl}$ crystal structure, h-ZrRuP crystallizes in the $\mathrm{P} \overline{6} 2 \mathrm{~m}$ space group with Wyckoff positions of $3(\mathrm{f})\left(x_{\mathrm{Zr}}\right.$, $0,0)$ for three $\mathrm{Zr}$ atoms, $3(\mathrm{~g})\left(x_{\mathrm{Ru}}, 0,1 / 2\right)$ for three $\mathrm{Ru}$ atoms, 2(d) $(1 / 3,2 / 3,1 / 2)$ for two $\mathrm{P}(1)$ atoms, and 1 (a) $(0,0,0)$ for one $\mathrm{P}(2)$ atom. Therefore the hexagonal crystal structure of $\mathrm{ZrRuP}$ is rather simple with nine atoms (three formula units) in the primitive unit cell and two internal parameters $\left(x_{\mathrm{Zr}}\right.$ and $\left.x_{\mathrm{Ru}}\right)$ for the position of the $\mathrm{Zr}$ and $\mathrm{Ru}$ atoms. The orthorhombic phase of $\mathrm{ZrRuP}$ adopts the simple orthorhombic TiNiSi structure (space group Pnma) with four $\mathrm{Zr}$ atoms at 4(c) $\left(x_{\mathrm{Zr}}, 1 / 4, z_{\mathrm{Zr}}\right)$, four Ru atoms at 4 (c) $\left(x_{\mathrm{Ru}}, 1 / 4, z_{\mathrm{Ru}}\right)$, and four $\mathrm{P}$ atoms at $4(\mathrm{c})\left(x_{\mathrm{P}}, 1 / 4, z_{\mathrm{P}}\right)$. As a consequence, the orthorhombic phase of $\mathrm{ZrRuP}$ is rather complex than its hexagonal structure with twelve atoms (four formula units) in the primitive unit cell and six internal parameters $\left(x_{\mathrm{Zr}}, z_{\mathrm{Zr}}, x_{\mathrm{Ru}}\right.$, $z_{\mathrm{Ru}}, x_{\mathrm{P}}$, and $\left.z_{\mathrm{P}}\right)$ for the position of the $\mathrm{Zr}, \mathrm{Ru}$, and $\mathrm{P}$ atoms. At the beginning of our calculations, the full structural optimization for both phases of ZrRuP has been executed by making use of total energy minimization and zero force criteria in order to obtain their equilibrium volume, lattice parameters, and internal parameters. At the equilibrium volume, o- $\mathrm{ZrRuP}$ has a lower energy of $0.1 \mathrm{meV} /$ atom than h-ZrRuP. This very small energy difference explains why ZrRuP exhibits polymorphism. After identifying the equilibrium volume of both phases, we have computed the energy versus volume data for them. Then, these data are fitted to the Murnaghan equation of states [67] in order to get the bulk modulus $(B)$ and its pressure derivative $\left(B^{\prime}\right)$. The calculated lattice parameters, the internal parameters, the bulk modulus and its pressure derivative for both phases of $\mathrm{ZrRuP}$ are presented in Table I, together with available experimental data [27,29-31,33,41] and previous theoretical results [51] for comparison. In general, the calculated values of lattice parameters and internal parameters for both phases of ZrRuP harmonize with the measured [27,29$31,33,41]$ and previously calculated [51] values while the calculated values of $B$ and $B^{\prime}$ for both phases of $\mathrm{ZrRuP}$ are comparable with their previous theoretical values [51]. In particular, when comparing our results for h-ZrRuP, the calculated lattice parameters $a$ and c vary from their measured ones $[27,29,41]$ within $1.0 \%$ and $2.0 \%$, respectively. For o$\mathrm{ZrRuP}$, the variation from experimental data $[30,31,33]$ of $a$, $b$, and $c$ is around $1.0 \%, 1.0 \%$, and $0.5 \%$, respectively.

Figure 2(a) displays the examination of the band structure of h-ZrRuP based on our GGA calculations. According to the calculated band structure, h-ZrRuP exhibits a threedimensional metallic character with three dispersive energy band passing over the Fermi level along the each symmetry directions except the $A-H$ and $H-L$ symmetry directions. These crossing bands are shown with red, blue, and green solid lines in the Fig. 2(a). Along the $A-H$ and $H-L$ symmetry directions, valence and conduction bands are obviously separated from each other. Figure 2(b) presents the calculated total and partial density of states (DOS) for h-ZrRuP. There are several gripping features in the total DOS which can be 
TABLE I. The calculated values of lattice parameters, internal parameters, bulk modulus $(B)$, and its pressure derivative $\left(B^{\prime}\right)$ for hexagonal and orthorhombic $\mathrm{ZrRuP}$ and their comparison with existing experimental data and previous theoretical results.

\begin{tabular}{|c|c|c|c|c|c|c|c|}
\hline Phase & $a(\AA)$ & $b(\AA)$ & $c(\AA)$ & $\left(x_{\mathrm{Zr}}, x_{\mathrm{Ru}}, x_{\mathrm{P}}\right)$ & $\left(z_{\mathrm{Zr}}, z_{\mathrm{Ru}}, z_{\mathrm{P}}\right)$ & $\mathrm{B}(\mathrm{GPa})$ & $B^{\prime}$ \\
\hline o-ZrRuP & 6.479 & 3.907 & 7.364 & $(0.0211,0.1503,0.2767)$ & $(0.6802,0.0611,0.3789)$ & 208.3 & 4.55 \\
\hline Experimental [30] & 6.417 & 3.862 & 7.322 & $(0.0225,0.1508,0.2744)$ & $(0.6804,0.0606,0.3778)$ & & \\
\hline Experimental [33] & 6.401 & 3.868 & 7.320 & & & & \\
\hline OLCAO-LDA [51] & & & & & & 235.2 & 3.84 \\
\hline h-ZRuP & 6.463 & & 3.858 & 0.5825 & 0.2455 & 210.4 & 3.59 \\
\hline Experimental $[27,29]$ & 6.459 & & 3.778 & 0.5854 & 0.2354 & & \\
\hline Experimental [41] & 6.445 & & 3.765 & & & & \\
\hline OLCAO-LDA [51] & 6.440 & & 3.765 & 0.5851 & 0.2447 & 237.9 & 2.95 \\
\hline
\end{tabular}

better explained by analyzing the partial DOS. At first glance, one can see a strong peak at $-11.5 \mathrm{eV}$ which is formed by $\mathrm{P} 3 s$ states with lesser but respectable contributions from $\mathrm{Zr}$ and $\mathrm{Ru}$ electronic states. This peak is clearly separated by a

(a)

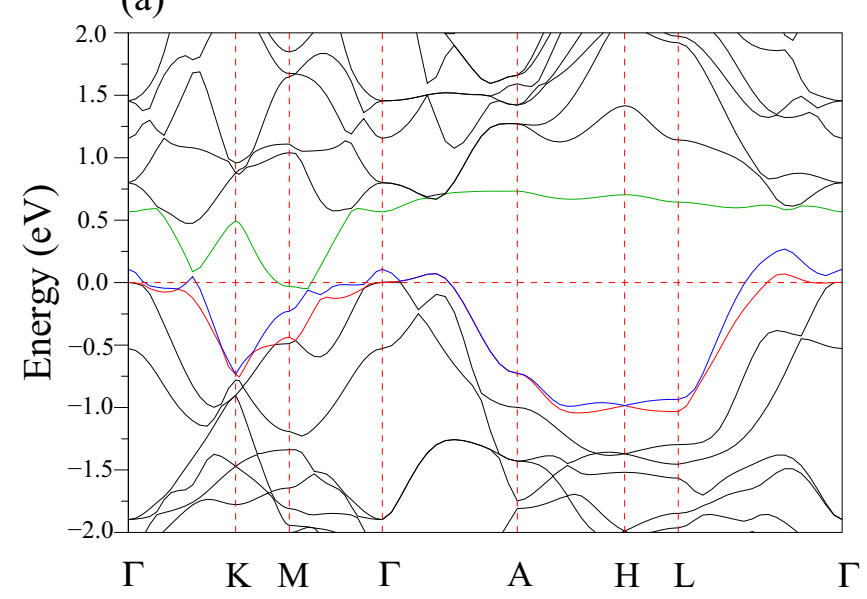

(b)

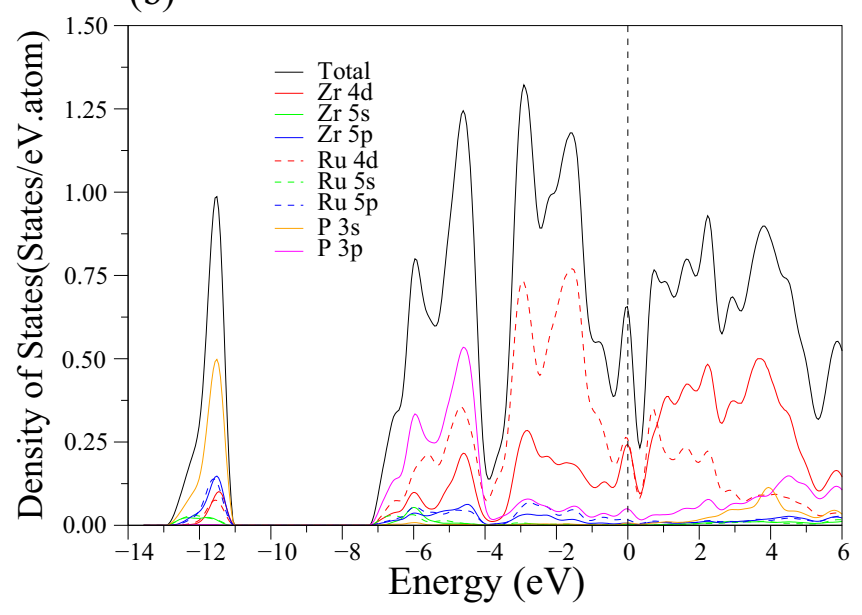

FIG. 2. (a) The calculated electronic band structure of h-ZrRuP along the high-symmetry lines in the first Brillouin zone of hexagonal lattice. The bands crossing the Fermi level are shown with red, blue, and green solid lines. (b) The calculated total and partial electronic density of states for h-ZrRuP. large gap of $3.7 \mathrm{eV}$ from the main valence band region which extends from -7.3 to $0 \mathrm{eV}$ (the Fermi level). $\mathrm{P} p, \mathrm{Ru} d$, and $\mathrm{Zr} d$ states display significant hybridization between each other under the energy range -7.3 to $-3.9 \mathrm{eV}$. The energy range from $-3.9 \mathrm{eV}$ to the Fermi level is predominated by the d states of transition metal atoms with lesser contributions from $\mathrm{P} 3 p, \mathrm{Ru} 5 p$, and $\mathrm{Zr} 5 p$ states. Since electrons close to the Fermi level are liable for superconductivity, the $N\left(E_{F}\right)$ is one of the important key quantities to analyze the origin of superconductivity in metallic materials. In agreement with previous theoretical studies [49,51], the Fermi level almost lies on the top of a narrow peak, which leads to a high $N\left(E_{F}\right)$ value of 0.61 states $/ \mathrm{eV}$ per atom. It is worth to mention that this value is smaller than the FLAPW value [49] 0.71 states/eV per atom but higher than the OLCAO value [51] of 0.44 states/eV per atom. The largest contribution to $N\left(E_{F}\right)$ is made by the $4 d$ states of Ru atoms within approximately $43 \%$ $\left[N^{\mathrm{Ru}(4 d)}\left(E_{F}\right)=0.26\right.$ states $/ \mathrm{eV}$ per atom], the second largest contribution to $N\left(E_{F}\right)$ comes from the $4 d$ states of $\mathrm{Zr}$ atoms within approximately $41 \%\left[N^{\mathrm{Zr}(4 d)}\left(E_{F}\right)=0.25\right.$ states $/ \mathrm{eV}$ per atom]. Therefore the contribution from $\mathrm{Zr}$ atoms is comparable to that from $\mathrm{Ru}$ atoms but the contribution from $\mathrm{P}$ atoms is much smaller as compared to the contributions from $\mathrm{Zr}$ or $\mathrm{Ru}$ atoms within approximately $11 \%\left[N^{P}\left(E_{F}\right)=0.07\right.$ states $/ \mathrm{eV}$ per atom]. Since ruthenium and zirconium $d$ electrons largely contribute to the DOS at the Fermi level, they must play the dominant role in the electrical transport.

The electronic band structure, and total and partial DOS for o-ZrRuP are shown in Fig. 3. The overall band profile accords with previous theoretical results [51]. The electronic structure diagram also suggests the metallic character of this phase since up to four bands cross the Fermi level. These bands are shown in different colors in Fig. 3(a). Each pair of these Fermi crossing bands are shown degeneracy along the $X-S, Z-U$, and $R-T$ directions. Although the DOS features of o-ZrRuP are similar to those of $h-Z r R u P$, the bands at the Fermi level are more dispersive than those in the hexagonal phase which gives rise to a lower $N\left(E_{F}\right)$ value of 0.54 states $/ \mathrm{eV}$ per atom. The value of $N\left(E_{F}\right)$ for o-ZrRuP consists of around $9 \% \mathrm{P}$ electronic states $\left[N\left(E_{F}^{P}\right)=0.05\right.$ states $/ \mathrm{eV}$ per atom $], 43 \% \mathrm{Zr}$ electronic states $\left[N\left(E_{F}^{\mathrm{Zr}}\right)=0.23\right.$ states $/ \mathrm{eV}$ per atom] and $48 \%$ $\mathrm{Ru}$ electronic states $\left[N\left(E_{F}^{R u}\right)=0.26\right.$ states/eV]. In particular, $\mathrm{Zr} 4 d$ and $\mathrm{Ru} 4 d$ states alone contribute to $N\left(E_{F}\right)$ up to $40 \%$ 
(a)

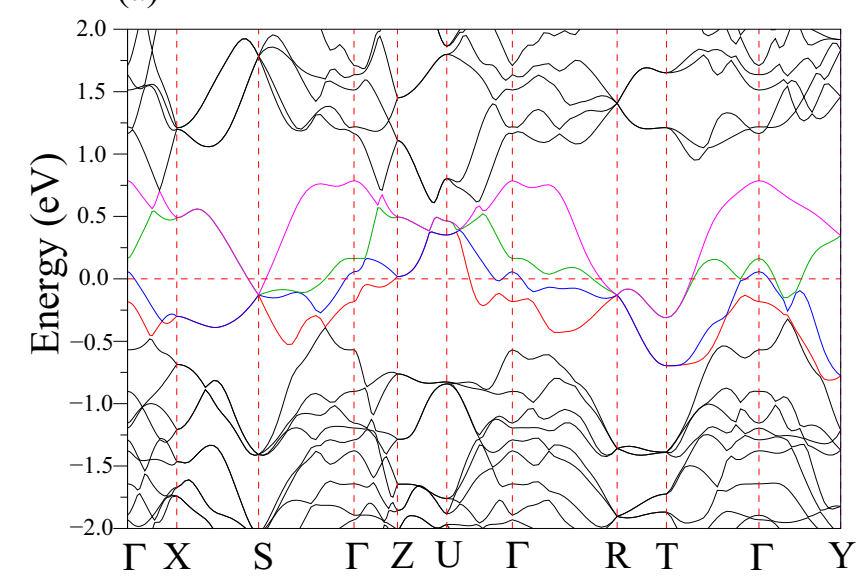

(b)

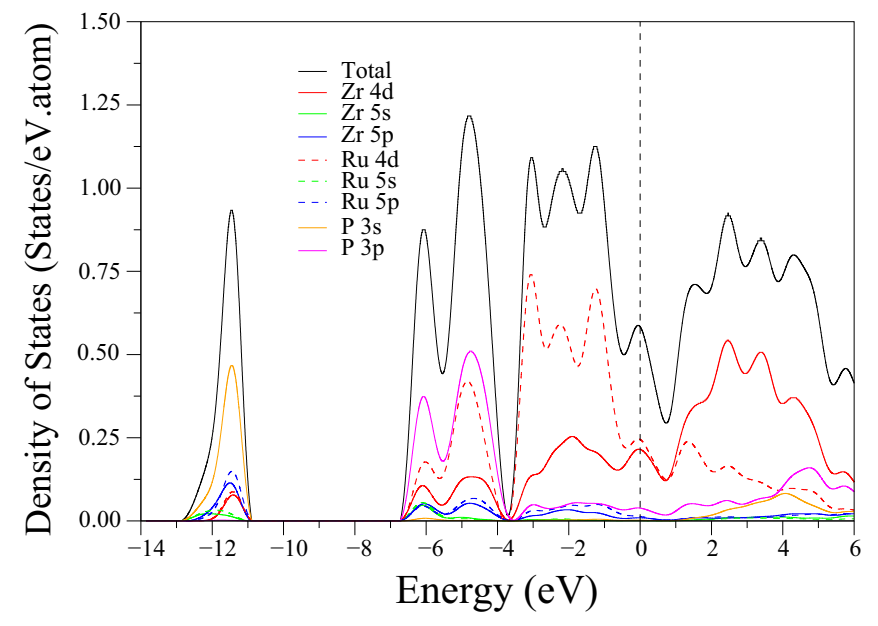

FIG. 3. (a) The calculated electronic band structure of o-ZrRuP along the high-symmetry lines in the first Brillouin zone of simple orthorhombic lattice. The bands crossing the Fermi level are shown with red, blue, green and magenta solid lines. (b) The calculated total and partial electronic density of states for o-ZrRuP.

and $44 \%$, respectively. Since the states near the Fermi level for both phases are mainly contributed by transition metal atoms, their $d$ states and vibrations must be the main actors of superconductivity in $\mathrm{h}-\mathrm{ZrRuP}$ and $\mathrm{o}-\mathrm{ZrRuP}$ according to the well-known BCS theory.

The calculated Fermi surface sheets for h-ZrRuP and oZrRuP are presented in Figs. 4(a) and 4(b), respectively. The h-ZrRuP compound has three Fermi surface sheets, and the bands that constituted these Fermi surfaces are shown with red, blue, and green solid lines in Fig. 2(a), respectively. On the other hand, the o-ZrRuP compound has four different Fermi surface sheets, and the corresponding energy bands are presented in Fig. 3(a) with red, blue, green, and magenta colors, respectively. These Fermi surface results are in good accordance with previous Fermi surface calculations $[49,50]$. In Fig. 4(a), the first two Fermi surfaces are mainly have hole characteristics and have a symmetric nesting around the $\Gamma$ high-symmetry point. These two surfaces are constructed by the electronic bands lying around the Fermi energy level along The $\Gamma-K, M-\Gamma, \Gamma-A$, and $L-\Gamma$ directions as can be (a)
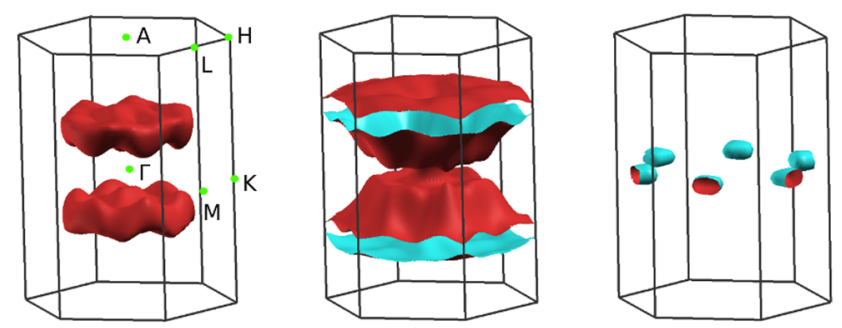

(b)
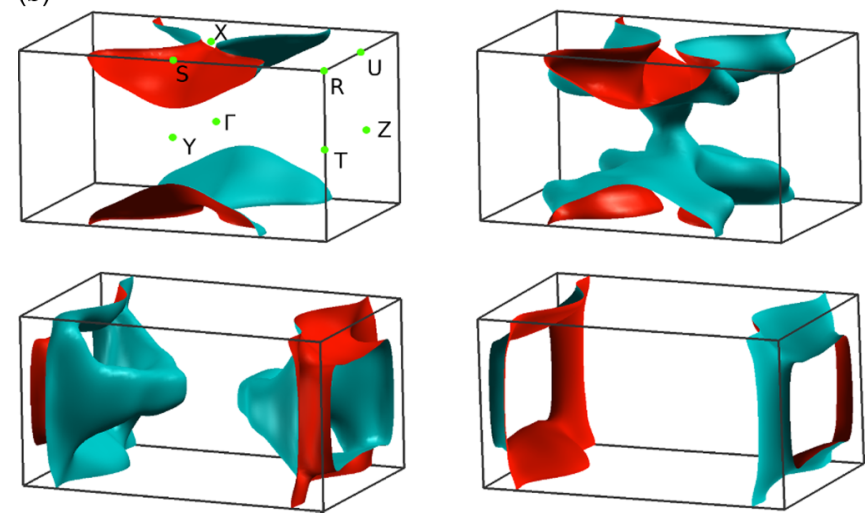

FIG. 4. The calculated Fermi surfaces for (a) h-ZrRuP and (b) oZrRuP.

seen from the Fig. 2(a). On the other hand, the third Fermi surface in Fig. 4(a) is consisted from the electrons and formed a closed surface around the $M$ symmetry point. For o-ZrRuP compound, the first two Fermi surfaces are shown hole characteristics and the last two ones are shown electron characteristics that presented in Fig. 4(b). While the first Fermi surface formed a bow-tie shape around the $Z$ point, second Fermi surface makes an hollow tube which encircles the $\Gamma$ point. The third and the fourth Fermi surfaces are mainly constituted by the electronic bands which are lengthen along the $\Gamma-R, \Gamma-Y$, and $\Gamma-T$ as can be seen from Fig. 3(a).

\section{B. Elastic and mechanical properties}

The calculation of single-crystal elastic constants is mandatory for the further theoretical realizing of the generation of superconductivity as well as being substantial for its practical application. According to the BCS theory, the origin of superconductivity arises from the electron-lattice interaction. Since the long-wavelength phonon spectra depends on the elastic constants of the material, they should play a noteworthy role in the generation of superconducting state. For a hexagonal system, the number of independent singlecrystal elastic constants is five, namely, $\mathrm{C}_{11}, \mathrm{C}_{12}, \mathrm{C}_{13}, \mathrm{C}_{33}$, and $\mathrm{C}_{44}$. The sixth one $\mathrm{C}_{66}$ is given as $\mathrm{C}_{66}=\frac{1}{2}\left(\mathrm{C}_{11}-\mathrm{C}_{12}\right)$. For an orthorhombic system, there are nine independent elastic constants, namely, $\mathrm{C}_{11}, \mathrm{C}_{12}, \mathrm{C}_{13}, \mathrm{C}_{22}, \mathrm{C}_{23}, \mathrm{C}_{33}, \mathrm{C}_{44}, \mathrm{C}_{55}$, and $\mathrm{C}_{66}$. In this work, the well-known stress-strain method [54,55] is used in evaluating the single-crystal elastic constants of both phases. Table II presents our calculated results for the elastic constants of both phases. Unfortunately, no experimental as well as previous theoretical results on the elastic properties of both phases subsist yet. h-ZrRuP has the weaker 
TABLE II. The calculated values of independent elastic constants (in GPa) for orthorhombic and hexagonal phases of ZrRuP. For a hexagonal phase, there are five linearly independent single-crystal elastic constants, namely, $\mathrm{C}_{11}\left(=\mathrm{C}_{22}\right), \mathrm{C}_{12}, \mathrm{C}_{13}\left(=\mathrm{C}_{23}\right), \mathrm{C}_{33}$, and $\mathrm{C}_{44}\left(=\mathrm{C}_{55}\right)$. The sixth one $\mathrm{C}_{66}$ equals to $\frac{1}{2}\left(\mathrm{C}_{11}-\mathrm{C}_{12}\right)$.

\begin{tabular}{lcccccrrrr}
\hline \hline Source & $\mathrm{C}_{11}$ & $\mathrm{C}_{12}$ & $\mathrm{C}_{13}$ & $\mathrm{C}_{22}$ & $\mathrm{C}_{23}$ & $\mathrm{C}_{33}$ & $\mathrm{C}_{44}$ & $\mathrm{C}_{55}$ \\
\hline o-ZrRuP & 336.48 & 150.57 & 129.46 & 334.49 & 156.02 & 376.54 & 131.04 & 126.48 \\
h-ZrRuP & 352.96 & 134.74 & 176.05 & 352.96 & 176.05 & 323.73 & 87.04 & 87.04 & 114.81 \\
\hline \hline
\end{tabular}

resistance against the shear deformations since its $\mathrm{C}_{44}$ value is considerably lower than that of o-ZrRuP. For both phases, the values of $\mathrm{C}_{11}$ and $\mathrm{C}_{33}$ are higher than those of remaining elastic constants, which reveal that they are considerably incompressible under uniaxial stress along $\boldsymbol{x}$ or $\boldsymbol{z}$ axis. The mechanical stability criterions [68] for a hexagonal system (h-ZrRuP) and an orthorhombic system (o-ZrRuP) can be expressed as

\section{Hexagonal phase}

$$
C_{44}>0 ; \quad C_{11}>\left|C_{12}\right| ; \quad \text { and } \quad\left(C_{11}+2 C_{12}\right) C_{33}>2 C_{13}^{2} \text {. }
$$

\section{Orthorhombic phase}

$$
\begin{aligned}
& C_{i i}>0(i=1,6), \quad C_{11}+C_{22}-2 C_{12}>0, \\
& C_{22}+C_{33}-2 C_{23}>0, \quad C_{11}+C_{33}-2 C_{13}>0, \\
& C_{11}+C_{22}+C_{33}+2 C_{12}+2 C_{13}+2 C_{23}>0 .
\end{aligned}
$$

As can be derived from Table II, the calculated elastic constants of both phases fully satisfy their aforementioned mechanical stability conditions. Therefore we can verify that both phases of ZrRuP are mechanically stable in their crystal structures.

The polycrystalline bulk modulus $\left(B_{\mathrm{VRH}}\right)$ and the polycrystalline shear modulus $\left(G_{\mathrm{VRH}}\right)$ can be directly calculated from the single crystal elastic constants by the Voigt-ReussHill (VRH) approximations [56-58]. The Voigt approximation [56] refers to the upper bound of mechanical modulus in terms of the uniform strain while Reuss approximation [57] provides the lower limit in terms of the uniform stress. At the end, the Hill approximation [58] represents the average value of mechanical modulus between Voigt and Reuss approximations [56,57]. Then, the values of $B_{H}$ and $G_{H}$ can be used to determine the Young's modulus E and Poisson's ratio $\sigma$ by the help of following expressions:

$$
E=\frac{9 G_{H} B_{H}}{\left(3 B_{H}+G_{H}\right)} \quad \text { and } \quad \sigma=\frac{3 B_{H}-2 G_{H}}{2\left(3 B_{H}+G_{H}\right)} .
$$

The calculated values of bulk modulus $B_{\mathrm{VRH}}$, shear modulus $G_{\mathrm{VRH}}$, Young's modulus E, $B_{H} / G_{H}$ ratio and Poisson's ratio $(\sigma)$ for both phases of $\mathrm{ZrRuP}$ are listed in Table III. Unfortunately, no experimental as well as previous theoreti- cal results on the elastic moduli of both phases are present yet. However, the calculated $B_{H}$ values of both phases are comparable with their corresponding values determined from total energy calculations (see also Table I), indicating the quality of our GGA results. Bulk modulus can be used as an initiatory measure of the hardness of the material but in order to promote it, other mechanical properties must be considered. A high bulk modulus does not always refer to a harder material since shear modulus may even give a better connection with hardness. Although the bulk modulus values of h-ZrRuP are slightly larger than those of o-ZrRuP, its shear modulus values are considerably lower than those of o-ZrRuP. This observation reveals that o-ZrRuP is harder than h-ZrRuP. Furthermore, the Young's modulus $E$ is usually connected to the stiffness of material. If its value is large, the material becomes stiffer. According to Table III, o-ZrRuP is $20 \%$ stiffer than h-ZrRuP. According to Pugh's criteria [69], if the $B_{H} / G_{H}$ ratio is larger than its limit value of 1.75 , the material acts in a ductile manner; otherwise its character is brittle. Table III reveals that both phases of $\mathrm{ZrRuP}$ are ductile in nature but the hexagonal one has a better ductility than the orthorhombic one. The value of Poisson's ratio amounts to around 0.1 in covalent materials and around 0.33 in metallic materials [70]. Therefore Table III indicates that $\mathrm{h}-\mathrm{ZrRuP}$ is soft (ductile) due to strong metallic bonding. For o-ZrRuP, the lower value of Poisson's ratio proposes that this phase may have the existence of short directional covalent bonds with metallic bonds in nature. The above discussions suggest that the lattice of $\mathrm{h}-\mathrm{ZrRuP}$ is considerably softer than that of $\mathrm{o}-\mathrm{ZrRuP}$. The softer lattice of $\mathrm{h}-\mathrm{ZrRuP}$ as compared to that of o-ZrRuP can make its $T_{c}$ value much higher than that of o-ZrRuP. The McMillan-Hopfield expression [71,72] gives the electron-phonon coupling parameter $(\lambda)$ as

$$
\lambda=\frac{N\left(E_{F}\right)\left\langle I^{2}\right\rangle}{M\left\langle\omega^{2}\right\rangle} .
$$

Where $\left\langle I^{2}\right\rangle$ refers to the averaged square of the electronphonon matrix and the $\mathrm{M}\left\langle\omega^{2}\right\rangle$ refers to the dimension of the force constants. The soft lattice will make the values of force constants smaller, thereby increasing the value of $\lambda$ as well as the value of $T_{c}$. We will further discuss this in the next section. A favorable parameter to define the extent of anisotropy is

TABLE III. The calculated values of isotropic bulk modulus $B_{\mathrm{VRH}}$, shear modulus $G_{\mathrm{VRH}}$, Young's modulus $E$ (all in GPa), $B_{H} / G_{H}$ ratio, Poisson's ratio $(\sigma)$, the universal anisotropic index $\left(A^{u}\right)$, and percent anisotropy $\left(A_{B}, A_{G}\right)$ for orthorhombic and hexagonal phases of $\mathrm{ZrRuP}$.

\begin{tabular}{ccccccccccccc}
\hline \hline Source & $B_{V}$ & $B_{R}$ & $B_{H}$ & $G_{V}$ & $G_{R}$ & $G_{H}$ & $E$ & $B_{H} / G_{H}$ & $\sigma$ & $A^{u}$ & $A_{B}$ & $A_{G}$ \\
\hline o-ZrRuP & 213.29 & 212.78 & 213.03 & 115.23 & 113.16 & 114.19 & 290.65 & 1.866 & 0.273 & 0.0940 & 0.0010 & 0.0090 \\
h-ZrRuP & 222.59 & 222.52 & 222.55 & 92.83 & 90.526 & 91.66 & 241.82 & 2.428 & 0.319 & 0.1276 & 0.0002 & 0.0120 \\
\hline \hline
\end{tabular}


required for single crystals. Several anisotropic indexes are present to define the elastic anisotropy. In our calculations, we will use the universal anisotropic index $\left(A^{U}\right)$ and percent anisotropy $\left(A_{B}\right.$ and $\left.A_{G}\right)$ which have the following forms:

$$
\begin{gathered}
A^{U}=5 \frac{G_{V}}{G_{R}}+\frac{B_{V}}{B_{R}}-6 \geqslant 0, \\
A_{B}=\frac{B_{V}-B_{R}}{B_{V}+B_{R}}, \\
A_{G}=\frac{G_{V}-G_{R}}{G_{V}+G_{R}} .
\end{gathered}
$$

If these indexes equal to zero, the structure has an isotropic character; otherwise it has an anisotropic character. The calculated values of these anisotropic indexes are also given in Table III. The $A_{B}$ values of $\mathrm{h}-\mathrm{ZrRuP}$ and o-ZrRuP are smaller than their $A_{G}$ values, specifying that these phases are weakly anisotropic. Finally, the $A^{U}$ value of h-ZrRuP is significantly larger than that of o-ZrRuP. Therefore we can conclude that $\mathrm{h}-\mathrm{ZrRuP}$ has a stronger anisotropy character than o-ZrRuP.

The Debye temperature $\left(\Theta_{D}\right)$ is also an important quantity for a material which associated with thermal conductivity, specific heat, elastic constants, and superconductivity. At low temperatures, the vibration excitations merely originate from acoustic vibrations and therefore the value of $\Theta_{D}$ can be estimated from the value of average acoustic wave velocity $\left(V_{M}\right)$ from the following equation [73]:

$$
\Theta_{D}=\frac{h}{k_{B}}\left(\frac{3 n}{4 \pi} \frac{N_{A} \rho}{M}\right)^{1 / 3} V_{M},
$$

where $h, k_{B}, N_{A}, M, n$, and $\rho$ are Planck's constant, Boltzmann constant, Avogadro's number, the molecular weight, the number of atoms in the molecule, and the mass density, respectively. The values of acoustic wave velocities are given as [73]

$$
\begin{gathered}
V_{T}=\left(\frac{G_{H}}{\rho}\right)^{1 / 2}, \\
V_{L}=\left(\frac{3 B_{H}+4 G_{H}}{3 \rho}\right)^{1 / 2}, \\
V_{M}=\left[\frac{1}{3}\left(\frac{2}{V_{T}^{3}}+\frac{1}{V_{L}^{3}}\right)\right]^{-1 / 3},
\end{gathered}
$$

where $V_{T}, V_{L}$, and $V_{M}$ are transverse, longitudinal, and mean sound elastic wave velocities, respectively. The calculated values of $V_{T}, V_{L}, V_{M}$, and $\Theta_{D}$ for both phases of $\mathrm{ZrRuP}$ are presented in Table IV along with available experimental data for $\Theta_{D}$. This table shows that the acoustic waves of $\mathrm{o}-\mathrm{ZrRuP}$ are faster than those of $\mathrm{h}-\mathrm{ZrRuP}$ which makes its $\Theta_{D}$ value larger than that of h-ZrRuP. Usually, a high Debye temperature means a large thermal conductivity. Therefore we can conclude that o-ZrRuP has a better thermal conductivity as compared to h-ZrRuP. Finally, the lower $\Theta_{D}$ value of h$\mathrm{ZrRuP}$ confirms its soft character since the Debye temperature is also a good measure of lattice stiffness.
TABLE IV. The calculated values of transverse $\left(V_{T}\right)$, longitudinal $\left(V_{L}\right)$, mean sound elastic wave $\left(V_{M}\right)$ velocities, and Debye temperature $\left(\Theta_{D}\right)$ for orthorhombic and hexagonal phases of $\mathrm{ZrRuP}$.

\begin{tabular}{lcccc}
\hline \hline Source & $V_{T}(\mathrm{~m} / \mathrm{s})$ & $V_{L}(\mathrm{~m} / \mathrm{s})$ & $V_{M}(\mathrm{~m} / \mathrm{s})$ & $\Theta_{D}(\mathrm{~K})$ \\
\hline o-ZrRuP & 3789.13 & 6777.04 & 4218.01 & 503 \\
Experimental [28,31] & & & & 454 \\
h-ZrRuP & 3392.43 & 6579.01 & 3798.48 & 453 \\
Experimental [28,31] & & & & 345 \\
\hline \hline
\end{tabular}

\section{Phonons and electron-phonon interaction}

The primitive unit cell of o-ZrRuP involves 12 atoms; thus, it possesses 36 phonon branches for any chosen wave vector, out of that three acoustic and the rest of them are optical phonon branches. According to the point group symmetry of $\mathrm{D}_{2 h}$, the zone-center optical phonon modes of o-ZrRuP are represented as

$$
\begin{aligned}
\Gamma\left(D_{2 h}\right)= & 6 A_{g}+3 B_{1 g}+6 B_{2 g}+3 B_{3 g}+3 A_{u}+5 B_{1 u} \\
& +2 B_{2 u}+5 B_{3 u} .
\end{aligned}
$$

Both A and B modes are singly degenerate. The frequencies and electron-phonon coupling parameters $(\lambda)$ of zone-center phonon modes and the dominant ions in additive to the eigenvectors of vibrational modes for $\mathrm{o}-\mathrm{ZrRuP}$ are presented in Table V. This table obviously reveals that the zone-center $\lambda$ values of $\mathrm{o}-\mathrm{ZrRuP}$ are very low which may be one of the reasons behind of its low $T_{c}$ value. Even, the total value of these coupling parameters has a low value of 0.413 which also reveals a weak electron-phonon interaction in o-ZrRuP.

Since the primitive unit cell of $\mathrm{h}-\mathrm{ZrRuP}$ consists of nine atoms, there are 27 phonon vibrations, involving three acoustic ones and 24 optical ones. The zone-center optical phonon modes of this phase can be classified by the irreducible presentation of the point group $\mathrm{D}_{3 h}$ :

$$
\Gamma\left(D_{3 h}\right)=2 E^{\prime \prime}+6 E^{\prime}+2 A_{1}^{\prime}+A_{1}^{\prime \prime}+2 A_{2}^{\prime}+3 A_{2}^{\prime \prime}
$$

with the $\mathrm{A}$ and $\mathrm{E}$ modes being singly and doubly degenerate, respectively. The frequencies and electron-phonon coupling parameters of zone-center phonon modes and the dominant ions in additive to the eigenvectors of vibrational modes for $\mathrm{h}$ $\mathrm{ZrRuP}$ are presented in Table VI. The $\lambda$ of the lowest $E^{\prime \prime}$ mode is the largest among the zone-center phonon modes with a value of 0.239 . This can be connected to two reasons. First, the frequency of these phonon modes are the softest which may make their $\lambda$ value large according to the McMillan-Hopfield [see Eq. (1)]. Secondly, the atomic displacement pattern of this phonon mode is characterized by the vibrations of $\mathrm{Zr}$ and $\mathrm{Ru}$ atoms which dominate the density of states at the Fermi level with their $d$ states. During the calculation of total $\lambda$, the $\lambda$ values of $\mathrm{E}$ modes in Table VI must be multiplied by a factor of two due their double degeneracies. Therefore the total $\lambda$ value of zone-center phonon modes for $\mathrm{h}-\mathrm{ZrRuP}$ is found to be 1.186 which is much larger than the corresponding value of 0.413 for o-ZrRuP. This result indicates that electron-phonon interaction in $\mathrm{h}-\mathrm{ZrRuP}$ is much stronger than that in $\mathrm{o}-\mathrm{ZrRuP}$ which makes the $T_{c}$ value of $\mathrm{h}-\mathrm{ZrRuP}$ higher than that of o-ZrRuP. 
TABLE V. Calculated zone-center phonon frequencies $(v$ in THz) and their electron-phonon coupling parameters $(\lambda)$ and their eigen characters for ZrRuP with the orthorhombic TiNiSi-type structure. The notations of I, R, and S denote infrared active, Raman active and silent modes, respectively.

\begin{tabular}{|c|c|c|c|c|c|c|c|}
\hline Mode & $v$ & $\lambda$ & Eigencharacters & Mode & $v$ & $\lambda$ & Eigencharacters \\
\hline$A_{g}(\mathrm{R})$ & 4.39 & 0.016 & $\mathbf{Z r}+\mathrm{Ru}+\mathrm{P}$ & $B_{3 u}(\mathrm{I})$ & 4.40 & 0.038 & $\mathbf{Z r}+\mathrm{Ru}+\mathrm{P}$ \\
\hline$A_{u}(\mathrm{~S})$ & 4.61 & 0.006 & $\mathbf{Z r}+\mathrm{Ru}+\mathrm{P}$ & $A_{g}(\mathrm{R})$ & 4.67 & 0.013 & $\mathrm{Zr}+\mathrm{Ru}+\mathrm{P}$ \\
\hline$B_{3 g}(\mathrm{R})$ & 4.97 & 0.012 & $\mathbf{Z r}+\mathrm{Ru}+\mathrm{P}$ & $B_{3 u}(\mathrm{I})$ & 5.11 & 0.015 & $\mathrm{Zr}+\mathrm{Ru}+\mathrm{P}$ \\
\hline$B_{3 g}(\mathrm{R})$ & 5.28 & 0.010 & $\mathrm{Zr}+\mathbf{R u}+\mathrm{P}$ & $B_{2 u}(\mathrm{I})$ & 5.35 & 0.011 & $\mathrm{Zr}+\mathrm{Ru}$ \\
\hline$B_{1 g}(\mathrm{R})$ & 5.79 & 0.013 & $\mathbf{R u}+\mathrm{P}$ & $A_{g}(\mathrm{R})$ & 5.95 & 0.018 & $\mathrm{Zr}+\mathbf{R} \mathbf{u}+\mathrm{P}$ \\
\hline$B_{1 u}(\mathrm{I})$ & 5.96 & 0.004 & $\mathrm{Zr}+\mathrm{Ru}+\mathrm{P}$ & $B_{2 g}(\mathrm{R})$ & 5.98 & 0.020 & $\mathrm{Zr}+\mathrm{Ru}+\mathrm{P}$ \\
\hline$B_{3 u}(\mathrm{I})$ & 6.05 & 0.005 & $\mathbf{Z r}+\mathbf{R u}+\mathrm{P}$ & $B_{1 u}(\mathrm{I})$ & 6.62 & 0.005 & $\mathbf{Z r}+\mathrm{Ru}+\mathrm{P}$ \\
\hline$B_{2 g}(\mathrm{R})$ & 6.67 & 0.009 & $\mathrm{Zr}+\mathbf{R u}+\mathrm{P}$ & $B_{1 u}(\mathrm{I})$ & 9.05 & 0.026 & $\mathrm{Zr}+\mathrm{Ru}+\mathbf{P}$ \\
\hline$B_{1 u}(\mathrm{R})$ & 9.13 & 0.005 & $\mathrm{Zr}+\mathrm{Ru}+\mathbf{P}$ & $B_{2 g}(\mathrm{R})$ & 9.37 & 0.011 & $\mathrm{Zr}+\mathrm{Ru}+\mathbf{P}$ \\
\hline$B_{3 u}(\mathrm{I})$ & 9.41 & 0.008 & $\mathrm{Zr}+\mathrm{Ru}+\mathbf{P}$ & $A_{g}(\mathrm{R})$ & 9.44 & 0.007 & $\mathrm{Zr}+\mathrm{Ru}+\mathbf{P}$ \\
\hline
\end{tabular}

Although only zone-center phonon calculations are successful in explaining the large $T_{c}$ difference between two phases of ZrRuP, certain definition of the average value of $\lambda$ for the calculation of $T_{c}$ necessitates the understanding of full phonon spectra and phonon density of states. These physical quantities for o-ZrRuP are presented in Figs. 5(a) and 5(b), respectively. The phonon spectrum of o-ZrRuP contains only positive frequencies, revealing its dynamical stability in the orthorhombic TiNiSi-type crystal structure. This phonon spectra can be grouped in two explicit regions: Lowfrequency region (LFR) lengthening up 0.0 to $6.74 \mathrm{THz}$, and high-frequency region (HFR) between 8.66 and $11.60 \mathrm{THz}$. There is a gap of $1.92 \mathrm{THz}$ between these two regions due the mass difference between different types of atoms. LFR consists of three acoustic and 21 optical phonon branches while there are 12 optical phonon branches in the HFR. It is worth to mention that all phonon branches of o-ZrRuP display considerable dispersive character. The contributions of atomic vibrations to phonon bands can be much better discovered by examining the total and partial DOS. The DOS features below the gap region arise from the coupled vibrations of heavier $\mathrm{Zr}$ and $\mathrm{Ru}$ atoms with a smaller contribution from the lighter $\mathrm{P}$ atoms. The DOS features above the gap region are formed by the vibrations of lighter $\mathrm{P}$ atoms with negligible contributions from the remaining atoms.

The calculated phonon spectrum of h-ZrRuP is depicted along selected high-symmetry direction in the hexagonal Brillouin zone in Fig. 6(a) . At first glance, the phonon spectra of h-ZrRuP looks different from that of o-ZrRuP which can be related to the structural difference between these two phases. The phonon spectra of this phase can be divided into three regions by two narrow gaps of 0.14 and $0.62 \mathrm{THz}$. The first frequency region involves a set of 17 phonon branches lengthening up to $6.61 \mathrm{THz}$. The second region is the narrowest between 6.75 and $7.59 \mathrm{THz}$ and contains only one optical phonon branch. The remaining 9 phonon branches lie between 8.21 and $11.06 \mathrm{THz}$. Finally, it is worth to mention that the lower transverse acoustic $\left(\mathrm{TA}_{1}\right)$ branch of this phase is considerable softer than that of o-ZrRuP. We strongly believe that this softening may be crucial for the high $T_{c}$ value of $\mathrm{h}$-ZrRuP. In order to explore a connection between this soft character and electron-phonon interaction, we plot the

TABLE VI. Calculated zone-center phonon frequencies ( $v$ in THz) and their electron-phonon coupling parameters $(\lambda)$ and their eigen characters for $\mathrm{ZrRuP}$ with the hexagonal ZrNiAl-type structure The notations of I, R, and S denote infrared active, Raman active and silent modes, respectively.

\begin{tabular}{|c|c|c|c|c|c|c|c|}
\hline Mode & $v$ & $\lambda$ & Eigencharacters & Mode & $v$ & $\lambda$ & Eigencharacters \\
\hline$E^{\prime}(\mathrm{I}+\mathrm{R})$ & 4.24 & 0.076 & $\mathbf{Z r}+\mathrm{P}$ & $E^{\prime}(\mathrm{I}+\mathrm{R})$ & 5.00 & 0.038 & $\mathbf{Z r}+\mathrm{Ru}+\mathrm{P}$ \\
\hline$A_{1}^{\prime}(\mathrm{R})$ & 5.48 & 0.045 & $\mathbf{Z r}+\mathrm{Ru}$ & $A_{2}^{\prime}(\mathrm{S})$ & 5.92 & 0.019 & $\mathbf{Z r}+\mathrm{Ru}$ \\
\hline$E^{\prime}(\mathrm{I}+\mathrm{R})$ & 6.51 & 0.032 & $\mathrm{Zr}+\mathbf{R} \mathbf{u}+\mathrm{P}$ & $A_{1}^{\prime}(\mathrm{R})$ & 7.58 & 0.024 & $\mathrm{Zr}+\mathbf{R u}$ \\
\hline$E^{\prime}(\mathrm{I}+\mathrm{R})$ & 8.96 & 0.065 & $\mathrm{Zr}+\mathrm{Ru}+\mathbf{P}$ & $A_{1}^{\prime \prime}(\mathrm{S})$ & 9.19 & 0.019 & $\mathbf{P}$ \\
\hline
\end{tabular}


(a)

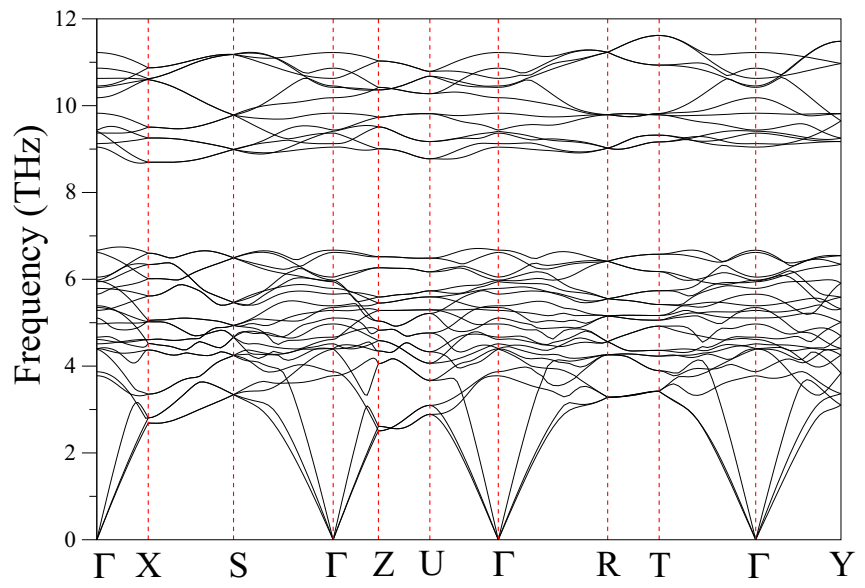

(b)

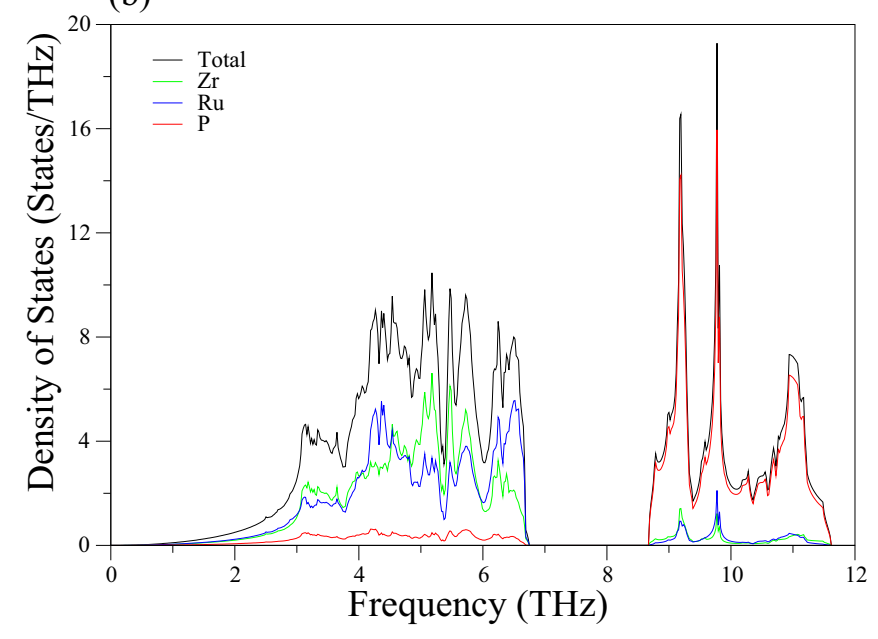

FIG. 5. (a) The calculated phonon spectrum of o-ZrRuP along the high-symmetry lines in the first Brillouin zone of simple orthorhombic lattice. (b) The calculated total and partial phonon density of states for o-ZrRuP.

dispersion of $\lambda$ for the $\mathrm{TA}_{1}$ branch of h-ZrRuP in Fig. 6(b). As can be seen from this figure, the $\lambda$ value of $\mathrm{TA}_{1}$ branch increases with changing $\boldsymbol{q}$ wave vector along the $\Gamma-K, M-K$, and $\Gamma-M$ symmetry directions. Therefore this observation reveals that the soft character of this phonon branch gives rise to strong electron-phonon interaction in $\mathrm{h}-\mathrm{ZrRuP}$. This conclusion is totally agreement with the conclusion made in the previous subsection. The total and partial DOS for h-ZrRuP are shown in Fig. 6(c). Although the electronic DOS of both phases are similar to each other, their phonon DOS look like different from each other. This observation supports the importance of their phonon properties for their large $T_{C}$ difference. Vibrations involving the three atomic species are present in the first frequency region. Ru vibrations dominate the second frequency region. The P-related phonon densities are quite dispersive, contributing to lattice vibrations over the full range of phonon frequencies because of their lighter masses than those of $\mathrm{Zr}$ and $\mathrm{Ru}$ atoms. In particular, the contribution of $\mathrm{P}$ atoms is strongest in the third frequency region. (a)

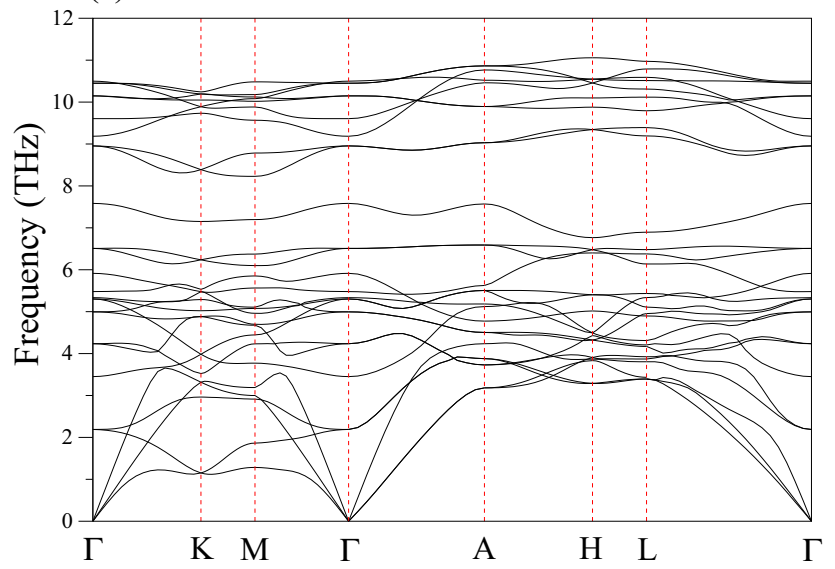

(b)

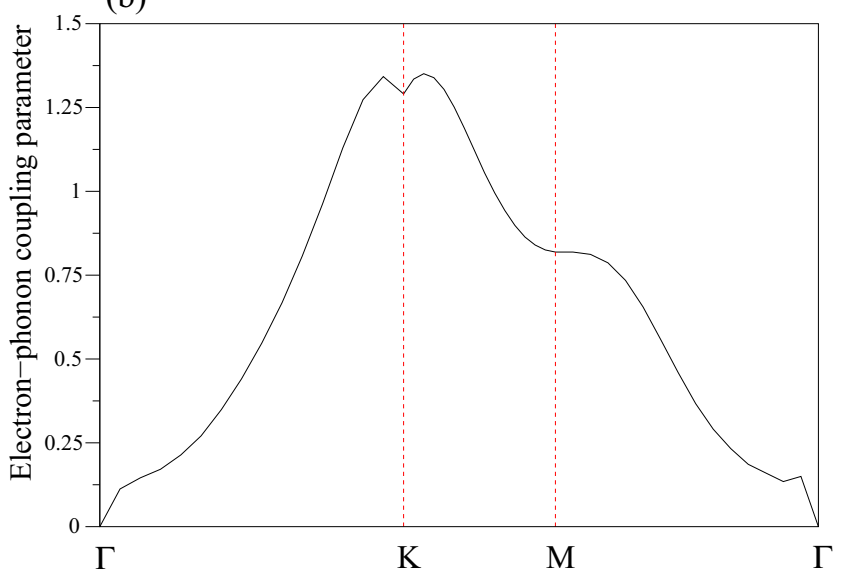

(c)

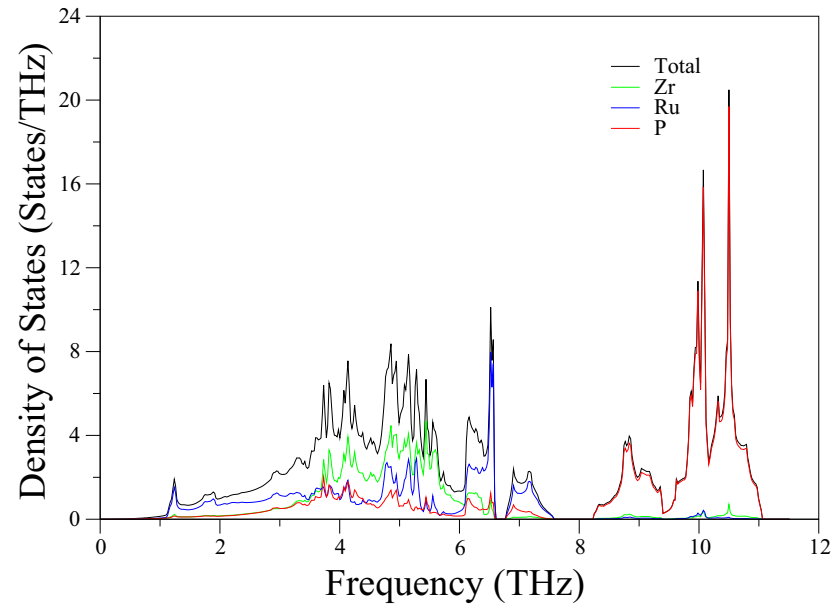

FIG. 6. (a) The calculated phonon spectrum of h-ZrRuP along the high-symmetry lines in the first Brillouin zone of hexagonal lattice. (b) The dispersion of electron-phonon coupling parameter for the lower transverse acoustic branch $\left(\mathrm{TA}_{1}\right)$ of h-ZrRuP along the $\Gamma-K-M-\Gamma$ directions. (c) The calculated total and partial phonon density of states for h-ZrRuP.

Since the calculated transverse and longitudinal acoustic (TA and LA) branches of both phases behave normally in the long-wave length limit with steep slopes, the values of 
some elastic constants can be obtained from the slopes of the acoustic branches in their phonon dispersion curves. For the orthorhombic phase, the acoustic velocity on the different directions can be given by the following equations [74]:

along the $\Gamma-X([100])$ direction:

$$
\begin{aligned}
{[100] V_{L A} } & =\sqrt{C_{11} / \rho}, \\
{[001] V_{T A_{1}} } & =\sqrt{C_{55} / \rho}, \\
{[010] V_{T A_{2}} } & =\sqrt{C_{66} / \rho} ;
\end{aligned}
$$

along the $\Gamma-Y([010])$ direction:

$$
\begin{aligned}
{[010] V_{L A} } & =\sqrt{C_{22} / \rho}, \\
{[100] V_{T A_{1}} } & =\sqrt{C_{66} / \rho}, \\
{[001] V_{T A_{2}} } & =\sqrt{C_{44} / \rho} ;
\end{aligned}
$$

along the $\Gamma-Z$ ([001]) direction:

$$
\begin{aligned}
{[001] V_{L A} } & =\sqrt{C_{33} / \rho}, \\
{[100] V_{T A_{1}} } & =\sqrt{C_{55} / \rho}, \\
{[010] V_{T A_{2}} } & =\sqrt{C_{44} / \rho} .
\end{aligned}
$$

By using above equations, the values of $\mathrm{C}_{11}, \mathrm{C}_{22}, \mathrm{C}_{33}, \mathrm{C}_{44}$, $\mathrm{C}_{55}$, and $\mathrm{C}_{66}$ are estimated to be 330.43, 285.64, 356.99, 125.77, 126.63, and 102.27 GPa which are comparable with their corresponding values obtained from the efficient stressstrain method calculations (see also Table II). It is worth to mention that the average values obtained from the different equations are used for $\mathrm{C}_{44}, \mathrm{C}_{55}$, and $\mathrm{C}_{66}$. For the hexagonal phase, the acoustic velocity on the different directions can be given by the following equations [75]:

along the $\Gamma-A$ ([001]) direction:

$$
[001] V_{L A}=\sqrt{C_{33} / \rho}, \quad[100] V_{T A}=\sqrt{C_{44} / \rho} ;
$$

along the $\Gamma-M([100])$ direction:

$$
\begin{aligned}
{[100] V_{L A} } & =\sqrt{C_{11} / \rho}, \\
{[010] V_{T A_{1}} } & =\sqrt{C_{66} / \rho}, \\
{[001] V_{T A_{2}} } & =\sqrt{C_{44} / \rho} .
\end{aligned}
$$

From the above equations, the values of $\mathrm{C}_{11}, \mathrm{C}_{33}, \mathrm{C}_{44}$, and $\mathrm{C}_{66}$ are estimated to be $340.90,327.79,74.92$, and $107.60 \mathrm{GPa}$ which lull with their corresponding values obtained from the efficient stress-strain method calculations (see also Table II). Once again, the average value is used for the $\mathrm{C}_{44}$ elastic constant. The above agreements supports the trustability of our GGA calculations.

The main objective of our work is to analyze the strengths of electron-phonon interaction in $\mathrm{h}-\mathrm{ZrRuP}$ and o-ZrRuP to better comprehend the large $T_{c}$ difference between them. For this cause, the frequency dependence of the Eliashberg spectral function $\alpha^{2} F(\omega)$ and the average electron-phonon coupling parameter $\lambda$ for both phases of $\mathrm{ZrRuP}$ are illustrated in Fig. 7. From this figure, we can notice that acoustic and low-frequency optical phonon modes of the hexagonal phase

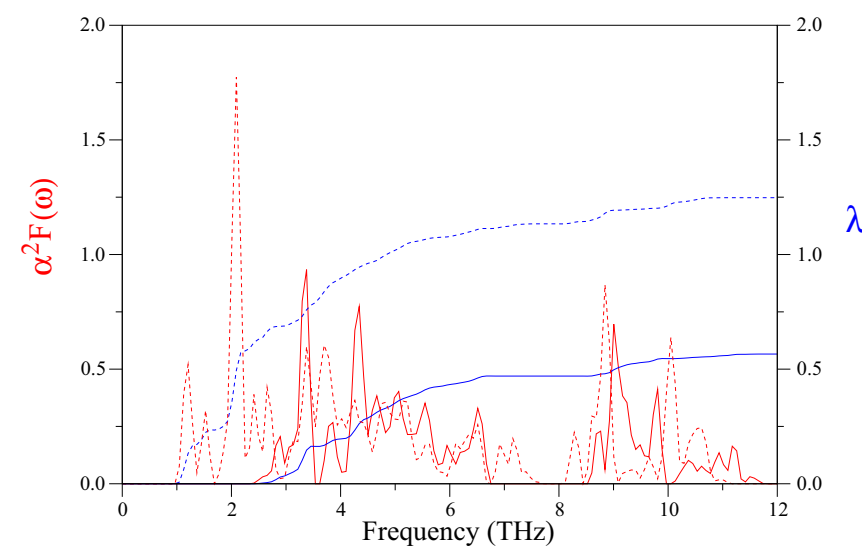

FIG. 7. The frequency variations of the Eliashberg spectral function $\alpha^{2} F(\omega)$ (red lines) and the average electron-phonon coupling parameter $\lambda$ (blue lines) for ZrRuP. Solid lines and dashed lines present our results for the orthorhombic and the hexagonal phases, respectively.

are relatively softer as compared to those of the orthorhombic phase. Furthermore, a comparison of the $\alpha^{2} F(\omega)$ and phonon DOS for h-ZrRuP reveals that the former is increased relative to latter primarily in the frequency region below $3.0 \mathrm{THz}$. In particular, a strong sharp peak at $2.0 \mathrm{THz}$ is present in the $\alpha^{2} F(\omega)$ of $\mathrm{h}-\mathrm{ZrRuP}$. The vibrational modes of $\mathrm{h}-\mathrm{ZrRuP}$ below $3.0 \mathrm{THz}$ offer about $56 \%$ to $\lambda$ which is more than the half of its total value. This large contribution proves that the phonon modes of h-ZrRuP with low-frequency (below 3.0 $\mathrm{THz}$ ) are strongly involved in the process of scattering of electrons. Phonon modes of h-ZrRuP between 3.0 and $7.6 \mathrm{THz}$ contribute about $35 \%$ to $\lambda$. Therefore we can imply that the medium-frequency phonon modes of $\mathrm{h}-\mathrm{ZrRuP}$ also couple considerably with the electrons at the Fermi level. Finally, the contribution of high-frequency phonon modes to $\lambda$ is about $9 \%$. For o-ZrRuP, its $\alpha^{2} F(\omega)$ reveals that its phonon branches below the gap region contribute strongly to $\lambda$ within $82 \%$. However, the phonon branches above the gap region contribute to $\lambda$ within $18 \%$. Both contributions are acceptable since the low-frequency phonon modes are dominated by the coupled vibrations of $\mathrm{Zr}$ and $\mathrm{Ru}$ atoms whereas the high-frequency optical modes are almost pure $\mathrm{P}$ states. A similar observation can be made for the hexagonal phase of $\mathrm{ZrRuP}$. Combining all of above results, we can reach this conclusion that the origin of high $T_{c}$ difference between $\mathrm{h}-\mathrm{ZrRuP}$ and o-ZrRuP arises from the relatively softer character of acoustic and low-frequency optical phonon modes of $\mathrm{h}-\mathrm{ZrRuP}$ as compared those of o-ZrRuP.

From the Eliashberg spectral function, we are able to derive the value of average electron-phonon coupling parameter

$$
\lambda=2 \int_{0}^{\infty} \frac{\alpha^{2} F(\omega)}{\omega} d \omega
$$

which gives $\lambda=1.25$ for $\mathrm{h}-\mathrm{ZrRuP}$ and $\lambda=0.57$ for $\mathrm{o}$ ZrRuP. These values signal that phonon and electron-phonon interaction properties are crucial in making $T_{c}$ much higher for $\mathrm{h}-\mathrm{ZrRuP}$ than o-ZrRuP. In our calculations, the superconducting temperature is estimated on the basis of the Allen-Dynes of the McMillan equation [71,72]: 
TABLE VII. The calculated values of physical quantities associated with superconductivity in h-ZrRuP and o-ZrRuP. Available experimental data and previous theoretical results are also included for comparison.

\begin{tabular}{|c|c|c|c|c|}
\hline Phase & $\begin{array}{c}N\left(E_{F}\right)(\text { states } / \mathrm{eV} \\
\text { per atom })\end{array}$ & $\omega_{\ln }(\mathrm{K})$ & $\lambda$ & $T_{c}(\mathrm{~K})$ \\
\hline h-ZrRuP & 0.61 & 138.02 & 1.25 & 12.49 \\
\hline Experimental $[27,32,38,39]$ & & & & 13.00 \\
\hline Experimental $[28,31]$ & 0.76 & & 0.79 & \\
\hline Experimental [29] & & & & 12.93 \\
\hline Experimental [35] & & & 1.10 & \\
\hline Experimental [41] & & & & 10.1 \\
\hline Experimental [42] & & & & 13.8 \\
\hline EHTBM [48] & 0.21 & & & \\
\hline FLAPW [49] & 0.71 & & & \\
\hline OLCAO-LDA [51] & 0.44 & & & \\
\hline o-ZrRuP & 0.54 & 231.62 & 0.57 & 3.89 \\
\hline Experimental $[28,31]$ & 0.72 & & 0.48 & 3.76 \\
\hline Experimental [30] & & & & 3.82 \\
\hline EHTBM [48] & 0.29 & & & \\
\hline FLAPW [50] & 0.71 & & & \\
\hline OLCAO-LDA [51] & 0.33 & & & \\
\hline
\end{tabular}

$$
\begin{aligned}
T_{c} & =\frac{\omega_{\ln }}{1.2} \exp \left(-\frac{1.04(1+\lambda)}{\lambda-\mu^{*}(1+0.62 \lambda)}\right), \\
\omega_{\ln } & =\exp \left(2 \lambda^{-1} \int_{0}^{\infty} \frac{d \omega}{\omega} \alpha^{2} F(\omega) \ln \omega\right),
\end{aligned}
$$

where $\omega_{\ln }$ is the logarithmically averaged phonon frequency and $\mu^{*}$ specifies the strength of the electron-electron interaction. Usually, $\mu^{*}$ has values between 0.10 and 0.16 [71,72]. In this work, its value is chosen to be 0.11 . The calculated values of the physical quantities $\left[N\left(E_{F}\right), \omega_{\ln }, \lambda\right.$, and $\left.T_{c}\right]$ relevant to superconductivity in both phases of $\mathrm{ZrRuP}$ are summarized in Table VII together with available experimental data $[27-32,35,38,39,41,42]$ and previous theoretical results [48-51]. The calculated values of $T_{c}(12.49 \mathrm{~K}$ for $\mathrm{h}$ $\mathrm{ZrRuP}$ and $3.89 \mathrm{~K}$ for o-ZrRuP) for both phases compare very well with their experimental data [27-32,35,38,39,41,42]. Our calculations suggest that the $N\left(E_{F}\right)$ value of $\mathrm{h}-\mathrm{ZrRuP}$ is slightly larger than that of o-ZrRuP while its $\omega_{\ln }$ value is much smaller than that of o-ZrRuP. This result is acceptable since both the soft mode and high electronic DOS at the Fermi level are positive to superconductivity. Although the soft mode gives rise to a smaller $\omega_{\ln }$, it makes a great contribution to $\lambda$. Therefore the large $\lambda$ value of $\mathrm{h}-\mathrm{ZrRuP}$ makes its $T_{c}$ value much higher than that of o-ZrRuP.

\section{SUMMARY}

We have researched the structural and electronic properties of hexagonal-ZrRuP (h-ZrRuP) and orthorhombic-ZrRuP (o-ZrRuP) by using the density functional theory with its generalized gradient approximation. The calculated values of lattice parameters and internal parameters for both phases of ZrRuP accord very well with existing experimental data.
Since the states near the Fermi level for both phases are strongly contributed by transition metal atoms, their $d$ states and vibrations must be the main actors of superconductivity. After obtaining the full relaxed geometry of both phases, their second order elastic constants have been estimated by using the efficient stress-strain method. The calculated values of elastic constants reveal the mechanical stability of $\mathrm{ZrRuP}$ in its hexagonal ZrNiAl-type and orthorhombic TiNiSi-type crystal structures. After obtaining second order elastic constants, the values of polycrystalline bulk modulus $(B)$, shear modulus $(G)$, Young's modulus $(E)$ and Poisson's ratio $(\sigma)$ for both phases have been calculated using the Voigt-Reuss-Hill approach. The calculated value of shear modulus $(G)$ and Young's modulus $(E)$ indicate that o-ZrRuP is stiffer than h$\mathrm{ZrRuP}$. According to Pugh's criteria, both phases of ZrRuP are ductile in nature but the hexagonal one has a better ductility than the orthorhombic one. In addition, the Poisson's ratio value of $\mathrm{h}-\mathrm{ZrRuP}$ confirms that this phase is soft (ductile) due to strong metallic bonding. Moreover, the lower Debye temperature $\left(\Theta_{D}\right)$ value of $\mathrm{h}-\mathrm{ZrRuP}$ also confirms its soft character since this temperature is a good measure of lattice stiffness. The above results demonstrate that the lattice of $\mathrm{h}-\mathrm{ZrRuP}$ is substantially softer than that of o-ZrRuP. The relatively softer lattice of $\mathrm{h}-\mathrm{ZrRuP}$ as compared to that of o-ZrRuP makes its $T_{c}$ value much higher than that of o-ZrRuP.

We have executed phonon calculations on the both phases of $\mathrm{ZrRuP}$ by the help of the linear response method. Our phonon results propose the dynamical stability of $\mathrm{ZrRuP}$ in its hexagonal and orthorhombic structures. Although the electronic DOS of both phases are similar to each other, their phonon DOS look like different from each other. This observation reveals the importance of their phonon properties for their large $T_{c}$ difference. A comparison of phonon dispersion curves for both phases indicates that the lower transverse acoustic branch of $\mathrm{h}-\mathrm{ZrRuP}$ is much softer than that of o-ZrRuP. The soft character of this phonon branch gives rise to strong electron-phonon interaction in $\mathrm{h}-\mathrm{ZrRuP}$. This conclusion is totally agreement with the conclusion made in the first paragraph. Since the calculated transverse and longitudinal acoustic branches of both phases behave normally in the long-wave limit with steep slopes, the values of some elastic constants can be determined from the slopes of the acoustic branches in their phonon dispersion curves. The calculated values of some elastic constants from the phonon dispersion curves of both phases harmonize with their corresponding values obtained from the efficient stress-strain method calculations. After phonon calculations, the linear response approach and the Migdal-Eliashberg approach have been combined to calculate the Eliashberg spectral function for both phases of $\mathrm{ZrRuP}$ which allows us to obtain their average electron-phonon coupling parameter $(\lambda)$ and logarithmic average of phonon frequency $\left(\omega_{\ln }\right)$. A comparison of their Eliashberg spectral functions reveals that acoustic and low-frequency optical phonon modes of the hexagonal phase are relatively softer as compared to those of the orthorhombic phase. These relatively softer phonon modes makes electronphonon interaction in $\mathrm{h}-\mathrm{ZrRuP}$ stronger than that in o-ZrRuP. The value of $\lambda$ is found to be 1.25 for $\mathrm{h}-\mathrm{ZrRuP}$ which much larger than the corresponding value of 0.57 for o-ZrRuP. Our electron-phonon interaction calculations reveal that the $N\left(E_{F}\right)$ 
value of h-ZrRuP is slightly larger than that of o-ZrRuP while its $\omega_{\text {ln }}$ value is much smaller than that of o-ZrRuP. Both the soft mode and high electronic DOS at the Fermi level are favorable to superconductivity. Although the soft mode leads to a smaller $\omega_{\mathrm{ln}}$, it makes a great contribution to $\lambda$. Therefore the large $\lambda$ value of $\mathrm{h}-\mathrm{ZrRuP}$ makes its superconducting transition temperature $\left(T_{c}\right)$ value much higher than that of o-ZrRuP. Finally, the value of $T_{c}$ is found to be $12.49 \mathrm{~K}$ for $\mathrm{h}-\mathrm{ZrRuP}$ and $3.89 \mathrm{~K}$ for o-ZrRuP which harmonize with their experimental values of 12.93 and $3.82 \mathrm{~K}$.
[1] C. B. Shoemaker and D. P. Shoemaker, A ternary alloy with $\mathrm{PbCl}_{2}$-type structure: TiNiSi(E), Acta Crystallogr. 18, 900 (1965).

[2] S. Rundqvist and P. C. Nawapong, The crystal structure of $\mathrm{ZrFeP}$ and related compounds, Acta Chem. Scand. 20, 2250 (1966).

[3] A. E. Dwight, M. H. Mueller, R. A. Conner, Jr., J. W. Downey, and $\mathrm{H}$. Knott, Ternary compounds with the $\mathrm{Fe}_{2} \mathrm{P}$-type structure, Trans. Met. Soc. AIME 242, 2075 (1968).

[4] N. Chaichit, P. Chalugune, S. Rukvichai, P. Choosang, V. Kaewchansilp, C.-O. Pontchour, P. Phavanantha, and S. Pramatus, Crystal structure refinements of $\mathrm{ZrCoP}, \mathrm{NbCoP}$, NbNiP and TaFeP, Acta Chem. Scand. A 32a, 309 (1978).

[5] W. Jeitschko and E. J. Reinbold, Structural chemistry of new scandium cobalt pho-sphides with a metal: Phosphorus ratio of 2:1, Z. Naturforsch. B 40, 900 (1985).

[6] G. A. Marking and H. F. Franzen, ZrNbP and HfNbP, new phases with the $\mathrm{Co}_{2} \mathrm{Si}$ structure, J. Alloys Compd. 204, L17 (1994).

[7] H. Kleinke and H. F. Franzen, Synthesis, crystal structure, and properties of $\mathrm{HfM}^{\prime} \mathrm{P}\left(\mathrm{M}^{\prime}=\mathrm{Fe}, \mathrm{Co}, \mathrm{Ni}\right)$ in comparison to ZrNiP, Z. Anorg. Allg. Chem. 622, 1893 (1996).

[8] H. Kleinke and H. F. Franzen, Sc-Sc bonding in the new ternary phosphide ScNiP, J. Solid State Chem. 137, 218 (1998).

[9] U. Pfannenschmidt, U. Ch. Rodewald, and R. Pöttgen, ScIrP with ZrNiAl-type Structure, Z. Naturforsch. B 66, 205 (2011).

[10] T. Dinges, M. Eul, and R. Pöttgen, TaRhGe with TiNiSi-type Structure, Z. Naturforsch. B 65, 95 (2010).

[11] T. Harmening, H. Eckert, C. M. Fehse, C. P. Sebastian, and R. Pöttgen, Sc-45 Solid State NMR studies of the silicides ScTSi ( $\mathrm{T}=\mathrm{Co}, \mathrm{Ni}, \mathrm{Cu}, \mathrm{Ru}, \mathrm{Rh}, \mathrm{Pd}, \mathrm{Ir}, \mathrm{Pt}$ ), J. Solid State Chem. 184, 3303 (2011).

[12] B. Heying, S. Haverkamp, U. Ch Rodewald, H. Eckert, S. C. Peter, and R. Pöttgen, The germanides $\mathrm{ScTGe}(\mathrm{T}=\mathrm{Co}, \mathrm{Ni}, \mathrm{Cu}$, $\mathrm{Ru}, \mathrm{Rh}, \mathrm{Pd}, \mathrm{Ag}, \mathrm{Ir}, \mathrm{Pt}, \mathrm{Au})$ - Structure and Sc-45 solid state NMR spectroscopy, Solid State Sci. 39, 15 (2015).

[13] R.-D. Hoffmann, Ute Ch. Rodewald, S. Haverkamp, C. Benndorf, H. Eckert, B. Heying, and R. Pöttgen, The hightemperature modification of ScRuSi Structure, 29Si and 45Sc solid state NMR spectroscopy, Solid State Sci. 72, 109 (2017).

[14] T. Kanomata, T. Kawashima, H. Utsugi, T. Goto, H. Hasegawa, and T. Kaneko, Magnetic-properties of the intermetallic compounds $\mathrm{MM}^{\prime} \mathrm{X}\left(\mathrm{M}=\mathrm{Cr}, \mathrm{Mn}, \mathrm{M}^{\prime}=\mathrm{Ru}, \mathrm{Rh}, \mathrm{Pd}\right.$, and $\mathrm{X}=\mathrm{P}$, As), J. Appl. Phys. 69, 4639 (1991).

[15] T. Eriksson, L. Bergqvist, T. Burkert, S. Felton, R. Tellgren, P. Nordblad, O. Eriksson, and Y. Andersson, Cycloidal magnetic order in the compound IrMnSi, Phys. Rev. B 71, 174420 (2005).

[16] Y.-K. Fang, J.-C. Yeh, W.-C. Chang, X.-M. Li, and W. Li, Structures, magnetic properties, and magnetocaloric effect in $\mathrm{MnCo}_{1-x} \mathrm{Ge}(0.02 \leqslant x \leqslant 0.2)$ compounds, J. Magn. Magn. Mater. 321, 3053 (2009).
[17] Z. Wang, Z. Nie, J. Zeng, R. Su, Y. Zhang, D. E. Brown, Y. Ren, and Y. Wang, First-order magnetostructural transformation in Fe doped Mn-Co-Ge alloys, J. Alloys Compd. 577, 486 (2013).

[18] G. J. Li, E. K. Liu, H. G. Zhang, Y. J. Zhang, J. L. Chen, W. H. Wang, H. W. Zhang, G. H. Wu, and S. Y. Yu, Phase diagram, ferromagnetic martensitic transformation and magnetoresponsive properties of Fe-doped MnCoGe alloys, J. Magn. Magn. Mater. 332, 146 (2013).

[19] J. W. Lai, Z. G. Zheng, R. Montemayor, X. C. Zhong, Z. W. Liu, and D. C. Zeng, Magnetic phase transitions and magnetocaloric effect of $\mathrm{MnCoGe}_{1-x} \mathrm{Si}_{x}$, J. Magn. Magn. Mater. 372, 86 (2014).

[20] S. C. Ma, D. Hou, F. Yang, Y. L. Huang, G. Song, Z. C. Zhong, D. H. Wang, and Y. W. Du, The antiferromagneticferromagnetic conversion and magnetostructural transformation in Mn-Ni-Fe-Ge ribbons, Appl. Phys. Lett. 104, 202412 (2014).

[21] S. C. Ma, Y. Su, M. Yang, F. Yang, Y. L. Huang, K. Liu, L. Zhang, and Z. C. Zhong, Magnetic phase transition and magnetocaloric effect in Mn-Fe-Ni-Ge ribbons, J. Alloys Compd. 629, 322 (2015).

[22] P. Dutta, S. Pramanick, S. Majumdar, D. Das, and S. Chatterjee, Multifunctional behavior of Fe-doped MnNiGe magnetic equiatomic compound, J. Magn. Magn. Mater. 395, 312 (2015).

[23] J. Liu, Y. Si, Y. Gong, G. Xu, Er Liu, F. Xu, and D. Wang, Enhanced magnetic refrigeration performance in metamagnetic MnCoSi alloy by high-pressure annealing, J. Alloys Compd. 701, 858 (2017).

[24] J. Q. Zhao, H. X. Zhu, C. L. Zhang, Y. G. Nie, H. F. Shi, E. J. Ye, Z. D. Han, and D. H. Wang, Magnetostructural transition and magnetocaloric effect in a MnCoSi-based material system, J. Alloys Compd. 735, 959 (2018).

[25] M. Onoue, R. Kobayashi, Y. Mitsui, M. Hiroi, K. Takahashi, A. Kondo, K. Kindo, Y. Uwatoko, and K. Koyama, Magnetic and structural properties of $\mathrm{MnCoGe}$ with minimal $\mathrm{Fe}$ and $\mathrm{Sn}$ substitution, Mater. Trans., 59, 1645 (2018).

[26] P. Bag and R. Nath, First order magneto-structural transition and magnetocaloric effect in MnNiGe0.9Ga0.1, Solid, State Commun. 270, 54 (2018).

[27] H. Barz, H. C. Ku, G. P. Meisner, Z. Fisk, and B. T. Matthias, Ternary transition metal phosphides: High-temperature superconductors, Proc. Natl. Acad. Sci. USA 77, 3132 (1980).

[28] G. R. Stewart, G. P. Meisner, and H. C. Ku, Superconductivity in $d$ - and f-Band Metal, edited by W. Buckel and W. Weber (Kernforschungszentrum, Karlsruhe, 1982), p. 331.

[29] G. P. Meisner and H. C. Ku, The superconductivity and structure of equiatomic ternary transition metal pnictides, Appl. Phys. A 31, 201 (1983).

[30] R. Muller, R. N. Shelton, J. W. Richardson, Jr., and R. A. Jacobson, Superconductivity and crystal structure of a new class of ternary transition metal phosphides $\mathrm{TT} / \mathrm{t}^{\prime} \mathrm{P}(\mathrm{TZr}, \mathrm{Nb}$, Ta and T/t'Ru, Rh), J. Less-Common Met. 92, 177 (1983). 
[31] H. Keiber, H. Wuhl, G. P. Meisner, and G. R. Stewart, Phonon anomalies in ZrRuP, Low. Temp. Phys. 55, 11 (1984).

[32] I. Shirotani, N. Ichihashi, K. Nozawa, M. Kinoshita, T. Yagi, K. Suzuki, and T. Enoki, Supercond. ZrRuP, Jpn. J. Appl. Phys. 32, 695 (1993).

[33] I. Shirotani, K. Tachi, N. Ichihashi, T. Adachi, T. Kikegawa, and O. Shimomura, Phase-transition of $\mathrm{ZrRuP}$ at high-temperatures and high-pressures, Phys. Lett. A 205, 77 (1995).

[34] I. Shirotani, K. Tachi, K. Takeda, S. Todo, T. Yagi, and K. Kanoda, Superconductivity of ZrRuSi prepared at highpressure, Phys. Rev. B 52, 6197 (1995).

[35] H. Salamati, F. S. Razavi, and G. Quirion, Effect of pressure on the superconductivity of ZrRuP, Physica C 292, 79 (1997).

[36] I. Shirotani, M. Hori, K. Tachi, S. Todo, and T. Yagi, Superconductivity of ZrRuGe with TiFeSi-type structure, J. Alloys Compd. 256, L1 (1997).

[37] I. Shirotani, K. Tachi, Y. Konno, S. Todo, and T. Yagi, Superconductivity of the ternary ruthenium compounds HfRuP and $\mathrm{ZrRuX}(\mathrm{X}=\mathrm{P}, \mathrm{As}, \mathrm{Si}$ or $\mathrm{Ge})$ prepared at a high pressure, Philos. Mag. B 79, 767 (1999).

[38] I. Shirotani, Y. Konno, D. Kato, C. Sekine, S. Todo, and T. Yaagi, Superconductivity of ternary equiatomic compounds with T-c of above 10 K, Jpn. J. Appl. Phys. 39, 525 (2000).

[39] I. Shirotani, D. Kato, A. Nishimoto, and T. Yagi, Superconductivity and crystal structure of ZrRu1-xRhxP alloys prepared at high pressure, J. Phys. Condens. Matter 13, 9393 (2001).

[40] I. Shirotani, Superconductivity of ternary metal compounds prepared at high pressures, Bull. Chem. Soc. Jpn. 76, 1291 (2003).

[41] R. E. Schaak and R. J. Cava, Boron substitution in ternary metal phosphide superconductors, Mater. Res. Bull. 39, 1231 (2004).

[42] L. M. Schoop, V. Ksenofontov, T. Gasi, R. J. Cava and C. Felser, The effect of Fe doping on superconductivity in $\mathrm{ZrRuP}$, Solid State Commun. 151, 1504 (2011).

[43] N. Kase, H. Suzuki, T. Nakano and N. Takeda, Superconductivity in the ternary silicide $\operatorname{TrIrSi}(\mathrm{Tr}=\mathrm{Ti}, \mathrm{Zr}$, and $\mathrm{Hf})$, Supercond. Sci. Technol. 29, 035011 (2016).

[44] Y. Okamoto, T. Inohara, Y. Yamakawa, and A. Yamakage, Superconductivity in the Hexagonal Ternary Phosphide ScIrP, J. Phys. Soc. Jpn. 85, 013704 (2016).

[45] T. Inohara, Y. Okamoto, Y. Yamakawa, and K. Takenaka, Synthesis and Superconducting Properties of a Hexagonal Phosphide ScRhP, J. Phys. Soc. Jpn. 85, 094706 (2016).

[46] B.-B. Ruan, X.-C. Wang, J. Yu, B.-J. Pan, Q.-G. Mu, T. Liu, G.-F. Chen, and Z. Ren, Superconductivity at $3.1 \mathrm{~K}$ in the orthorhombic ternary silicide ScRuSi, Supercond. Sci. Technol. 30, 025008 (2017).

[47] C. Benndorf, L. Heletta, G. Heymann, H. Huppertz, H. Eckert, and R. Pöttgen, NbOsSi and TaOsSi - Two new superconducting ternary osmium silicides, Solid State Sci. 68, 32 (2017);

[48] D. K. Seo, J. Ren, M. H. Whangbo, and E. Canadell, Electronic band structure study of the transport properties of the intermetallic compounds ZrRuP and ZrRuSi, Inorg. Chem. 36, 6058 (1997).

[49] I. Hase, Electronic structure of superconducting compounds hZrRuX (X = P, As, Si), Phys. Rev. B. 65, 174507 (2002).

[50] I. Hase, Electronic structure of the superconducting compounds o-ZrRuP and MoRuP, Phys. Rev. B. 68, 064506 (2003).

[51] W. Y. Ching, Y.-N. Xu, and L. Ouyang, Comparative study of the electronic structure of ternary superconductors MoRuP and $\mathrm{ZrRuP}$ in the orthorhombic and hexagonal phases, J. Appl. Phys. 93, 8209 (2003).

[52] P. Giannozzi, S. Baroni, N. Bonini, M. Calandra, R. Car, C. Cavazzoni, D. Ceresoli, G. L. Chiarotti, M. Cococcioni, I. Dabo, A. Dal Corso, S. de Gironcoli, S. Fabris, G. Fratesi, R. Gebauer, U. Gerstmann, C. Gougoussis, A. Kokalj, M. Lazzeri, L. Martin-Samos et al., QUANTUM ESPRESSO: A modular and open-source software project for quantum simulations of materials, J. Phys.: Condens. Matter 21, 395502 (2009).

[53] P. Giannozzi, O. Andreussi, T. Brumme, O. Bunau, M. Buongiorno Nardelli, M. Calandra, R. Car, C. Cavazzoni, D. Ceresoli, M. Cococcioni, N. Colonna, I. Carnimeo, A. Dal Corso, S. de Gironcoli, P. Delugas, R. A. DiStasio, Jr., A. Ferretti, A. Floris, G. Fratesi, G. Fugallo et al., Advanced capabilities for materials modeling with Quantum ESPRESSO, J. Phy.: Condens. Matter 29, 465901 (2017).

[54] L. Farst, J. M. Wills, B. Johansson, and E. Erikson, Elastic constants of hexagonal transtion metal, Phys. Rev. B 51, 17431 (1995).

[55] H. M. Tütüncü, H. Y. Uzunok, E. Karaca, S. Bağc1, and G. P. Srivastava, Ab initio investigation of spin orbit coupling effect on the physical properties of IrGe superconductor, Intermetallics 106, 107 (2019).

[56] W. Voigt, Lehrbuch der Kristallphysik (Springer Fachmedien Wiesbaden $\mathrm{GmbH}, 1928$ ).

[57] A. Reuss, Berechnung der Fließgrenze von Mischkristallen auf Grund der Plastizitätsbedingung für Einkristalle, Z. Angew. Math. Mech. 9, 49 (1929).

[58] R. Hill, The elastic behavior of a crystalline aggregate, Proc. Phys. Soc. Sect. A 65, 349 (1952).

[59] A. B. Migdal, Interaction between electrons and lattice vibrations in a normal metal, J. Exptl. Theoret. Phys. (U.S.S.R.) 34, 1438 (1958) [Sov. Phys. JETP 34, 996 (1958)].

[60] G. M. Eliashberg, Interaction between electrons and lattice vibrations in a superconductor, J. Exptl. Theoret. Phys. (U.S.S.R.) 38, 966 (1960) [Sov. Phys. JETP. 11, 696 (1960)].

[61] W. Kohn and L. J. Sham, Self-consistent equations including exchange and correlation effects, Phys. Rev. 140, A1133 (1965).

[62] J. P. Perdew, K. Burke, and M. Ernzerhof, Generalized Gradient Approximation Made Simple, Phys. Rev. Lett. 77, 3865 (1996).

[63] R. Stumpf, X. Gonze, and M. Scheffler, A List of Separable, Norm-conserving, Ab Initio Pseudopotentials (Fritz-HaberInstitut, Berlin, 1990).

[64] T. H. Fischer and J. Almlof, General methods for geometry and wave function optimization, J. Phys. Chem. 96, 9768 (1992).

[65] H. J. Monkhorst and J. D. Pack, Special points for Brillouinzone integrations, Phys. Rev. B 13, 5188 (1976).

[66] H. M. Tütüncü, H. Y. Uzunok, E. Karaca, G. P. Srivastava, S. Özer, and Ş. Uğur, Ab initio investigation of BCS-type superconductivity in $\mathrm{LuNi}_{2} B_{2} \mathrm{C}$-type superconductors, Phys. Rev. B 92, 054510 (2015).

[67] F. D. Murnaghan, The compressibility of media under extreme pressures, Proc. Natl. Acad. Sci. USA 30, 244 (1944).

[68] Z.-J. Wu, E.-J. Zhao, H.-P. Xiang, X.-F. Hao, and X.-J. Liu, J. Meng, Crystal structures and elastic properties of superhard 
$\mathrm{IrN}_{2}$ and $\mathrm{IrN}_{3}$ from first principles, Phys. Rev. B 76, 054115 (2007).

[69] S. F. Pugh, XCII. Relations between the elastic moduli and the plastic properties of polycrystalline pure metals, Phil. Mag. 45, 823 (1954).

[70] J. Haines, J. M. Leger, G. Bocquillon, Synthesis and design of superhard materials, Annu. Rev. Mater. Res. 31, 1 (2001).

[71] P. B. Allen and R. C. Dynes, Transition temperature of strongcoupled superconductors reanalyzed, Phys. Rev. B 12, 905 (1975).

[72] P. B. Allen and R. C. Dynes, Superconductivity at very strong coupling, J. Phys. C: Solid State Phys. 8, L158 (1975).
[73] O. L. Anderson, A simplified method for calculating the Debye temperature from elastic constants, J. Phys. Chem. Solids 24, 909 (1963).

[74] R. Li and Y. Duan, Structural and anisotropic elastic properties of hexagonal MP (M = Ti, Zr, Hf) monophosphides determined by first-principles calculations, Phil. Mag. A 96, 3654 (2016).

[75] K. Saramak, S. Limpijumnong, and W. L. Lambrecht, Pressuredependent elastic constants and sound velocities of wurtzite $\mathrm{SiC}, \mathrm{GaN}, \mathrm{InN}, \mathrm{ZnO}$, and CdSe, and their relation to the highpressure phase transition: A first-principles study, Phys. Rev. B 82, 035201 (2010). 